\title{
Finite affine planes in projective spaces
}

\author{
J. A.Thas H. Van Maldeghem \\ Ghent University, Belgium \\ $\{$ jat, hvm\}@cage . ugent. be
}

\begin{abstract}
We classify all representations of an arbitrary affine plane $\mathcal{A}$ of order $q$ in a projective space $\mathrm{PG}(d, q)$ such that lines of $\mathcal{A}$ correspond with affine lines and/or plane $q$-arcs and such that for each plane $q$-arc which corresponds to a line $L$ of $\mathcal{A}$ the plane of $\operatorname{PG}(d, q)$ spanned by the $q$-arc does not contain the image of any point off $L$ of $\mathcal{A}$.
\end{abstract}

\section{Introduction and Main Results}

The quadric Veronesean surface $\mathcal{V}_{2}^{4}$ over a finite field is a well known example of a representation of the finite projective plane $\mathrm{PG}(2, q)$ in a projective space $\mathrm{PG}(5, q)$ where the points of $\mathrm{PG}(2, q)$ are some points of $\mathrm{PG}(5, q)$, and the lines of $\mathrm{PG}(2, q)$ are certain conics. This also induces a representation of the affine plane $\operatorname{AG}(2, q)$ into $\mathrm{PG}(5, q)$. This time, the lines of $\mathrm{AG}(2, q)$ correspond to plane $q$-arcs in $\mathrm{PG}(5, q)$

Another well known representation of $\operatorname{AG}(2, q)$ using plane $q$-arcs as lines is an ovoid in $\mathrm{PG}(3, q)$ from which one point $p$ is removed, and the $q$-arcs arise as intersections of the ovoid with the non-tangent planes containing the point $p$. Instead of an ovoid, one can also use a hyperbolic quadric, or an oval cone, with appropriate point sets removed. But in these cases, some lines of $\mathrm{AG}(2, q)$ correspond with "affine lines" in $\mathrm{PG}(3, q)$.

The above representations all have the common property that, for each line $L$ of $\operatorname{AG}(2, q)$, the subspace of $\mathrm{PG}(d, q)$ ( $d=3$ or 5$)$ spanned by the points of the corresponding affine line or $q$-arc, does not contain any point of $\operatorname{AG}(2, q)$ off $L$. In the present paper, we take this as an axiom if the line $L$ corresponds to a plane $q$-arc and classify all representations 
of any affine plane of order $q$ in $\mathrm{PG}(d, q), d \geq 3$, with this property and for which lines correspond with affine lines and/or plane $q$-arcs of $\mathrm{PG}(d, q)$, for large enough $q$. The above well known examples of course appear, along with some new representations. Note that the case $d=2$ is trivial.

More exactly, we will prove the following theorem; in order to distinguish the lines of the affine plane from the lines of the ambient projective space, we call the former "blocks".

Main Result-Affine Planes. Let $\mathcal{A}=(\mathcal{P}, \mathcal{B})$ be an affine plane of order $q$ with point set $\mathcal{P}$ and set of blocks $\mathcal{B} \subseteq 2^{\mathcal{P}}$. Assume that

(i) the set $\mathcal{P}$ is a point set of $\mathrm{PG}(d, q), d \geq 3$ and $q \geq 7$, with $\langle\mathcal{P}\rangle=\mathrm{PG}(d, q)$;

(ii) the elements of $\mathcal{B}$ are either affine lines or plane $q$-arcs of $\mathrm{PG}(d, q)$;

(iii) if $L \in \mathcal{B}$ is a plane q-arc, then $\langle L\rangle \cap \mathcal{P}=L$.

Then exactly one of the following possibilities occurs.

(a) $d=5$. Here, the set $\mathcal{P}$ is the Veronesean surface $\mathcal{V}_{2}^{4}$ where a given conic $C$ is removed. The elements of $\mathcal{B}$ are the sets $D \cap \mathcal{P}$, with $D$ any conic on $\mathcal{V}_{2}^{4}$ distinct from $C$.

(b) $d=3$. Exactly one of the following cases occurs.

(b1) The set $\mathcal{P}$ is an ovoid $\mathcal{O}$ of $\mathrm{PG}(3, q)$ where one point $p \in \mathcal{O}$ is removed. The elements of $\mathcal{B}$ are the sets $D \backslash\{p\}$, with $D$ any oval on $\mathcal{O}$ through $p$.

(b2) The set $\mathcal{P}$ is a cone $\mathcal{K}$ with vertex $x$ and base an oval $O$, where we remove one generator $L$. There is a point $p$ on $L$ such that every $q$-arc on $\mathcal{P}$ arising from a plane intersection of $\mathcal{K}$ with a plane through $p$ is a block of $\mathcal{A}$, and the other blocks are the affine lines which are the intersections of $\mathcal{P}$ with the generators of $\mathcal{K}$ distinct from $L$.

(b3) The set $\mathcal{P}$ is a hyperbolic quadric $\mathcal{H}$ with two intersecting generators $L, M$ removed, $L \cap M=\{p\}$. The blocks are the $q$-arcs arising from plane intersections of $\mathcal{H}$ with planes through $p$ not tangent to $\mathcal{H}$, and the intersections of $\mathcal{P}$ with the generators of $\mathcal{H}$ distinct from $L$ and $M$.

(b4) The prime power $q$ is even and the set $\mathcal{P}$ is a cone $\mathcal{K}$ with vertex $p$ and base an oval $O$, where we remove $O$. The blocks are the q-arcs arising from plane intersections of $\mathcal{K}$ with planes not containing $O$ through the nucleus of $O$ and not passing through $p$, and the intersections of $\mathcal{P}$ with the generators of $\mathcal{K}$. 
(c) $d=4$. Exactly one of the following cases occurs.

$(c 1)$ Let $\mathcal{S}$ be a rational normal cubic scroll with directrix line $V$ and let $W$ be a given generator. Then $\mathcal{P}=\mathcal{S} \backslash(V \cup W)$, every affine line which is the intersection of $\mathcal{P}$ with a generator of $\mathcal{S}$ distinct from $W$ belongs to $\mathcal{B}$, and the other members of $\mathcal{B}$ are the plane $q$-arcs arising from the intersection of $\mathcal{P}$ with the non-singular conics on $\mathcal{S}$.

(c2) Let $\pi$ be a conic plane of the Veronesean surface $\mathcal{V}_{2}^{4}$ in $\mathrm{PG}(5, q)$, set $C=\pi \cap \mathcal{V}_{2}^{4}$, and let $p \in \pi \backslash C$, with $p$ not the nucleus of $C$ if $q$ is even. Let $\mathrm{PG}(4, q)$ be a hyperplane of $\mathrm{PG}(5, q)$ not containing $p$. Then $\mathcal{P}$ is the projection of $\mathcal{V}_{2}^{4} \backslash C$ from $p$ into $\mathrm{PG}(4, q)$. The set $\mathcal{B}$ does not contain any affine line and the plane $q$-arcs in $\mathcal{B}$ are the projections of $E \backslash C$, with $E$ ranging over the conics of $\mathcal{V}_{2}^{4}$ distinct from $C$.

For $q$ even, this defines a unique model; for $q$ odd, this defines exactly two projectively nonequivalent models, and the two cases correspond to whether $p$ is an interior or exterior point for $C$.

(c3) Here, $q$ is even, and coordinates can be chosen such that the set $\mathcal{P}$ consists of all points with coordinates $\left(x_{0}, x_{1}, x_{2}, x_{3}, x_{4}\right)$, where

$$
\left\{\begin{array}{l}
x_{0}=a^{\sigma}, \\
x_{1}=b^{\sigma}, \\
x_{2}=c^{\sigma}, \\
x_{3}=a c^{\sigma-1}, \\
x_{4}=b c^{\sigma-1},
\end{array}\right.
$$

with $a, b, c \in \mathrm{GF}(q), c \neq 0$, and $\sigma$ an automorphism which generates $\operatorname{AutGF}(q)$. The set $\mathcal{B}$ does not contain any affine line, and the $q^{2}+q$ plane $q$-arcs of $\mathcal{B}$ are all plane q-arcs lying in $\mathcal{P}$.

For $\sigma=2$, the affine plane $\mathcal{A}$ can also be described as in (c2) above, but with $p$ being the nucleus of $C$.

(c4) Here, $q$ is even, and coordinates can be chosen such that the set $\mathcal{P}$ consists of all points with coordinates $\left(x_{0}, x_{1}, x_{2}, x_{3}, x_{4}\right)$, where

$$
\left\{\begin{array}{l}
x_{0}=a^{\sigma} \\
x_{1}=b^{\sigma} \\
x_{2}=c^{\sigma} \\
x_{3}=a c^{\sigma-1} \\
x_{4}=b c^{\sigma-1}
\end{array}\right.
$$


with $a, b, c \in \mathrm{GF}(q), b \neq 0$, and $\sigma$ an automorphism which generates $\operatorname{AutGF}(q)$ (the expression $0^{\sigma-1}$ equals 0 ). The set $\mathcal{B}$ contains exactly one affine line, which is the affine line with equations

$$
\left\{\begin{array}{r}
X_{2}=X_{3}=X_{4}=0 \\
X_{1} \neq 0
\end{array}\right.
$$

which corresponds to $c=0$, and the $q^{2}+q-1$ plane $q$-arcs of $\mathcal{B}$ are all plane $q$-arcs lying in $\mathcal{P}$.

For $\sigma=2$, the affine plane $\mathcal{A}$ can also be described as follows. Let $\mathcal{V}_{2}^{4}$ be the Veronesean surface in $\mathrm{PG}(5, q)$, q even, and let $\mathcal{N}$ be the nucleus plane of $\mathcal{V}_{2}^{4}$. Let $p \in \mathcal{N}$ and let $\mathrm{PG}(4, q)$ be a hyperplane of $\mathrm{PG}(5, q)$ not containing $p$. Let $C$ be the conic on $\mathcal{V}_{2}^{4}$ with nucleus $p$ and let $D$ be some conic on $\mathcal{V}_{2}^{4}$ distinct from $C$. Then $\mathcal{P}$ is the projection of $\mathcal{V}_{2}^{4} \backslash D$ from $p$ into $\mathrm{PG}(4, q)$. The projection of $C \backslash D$ is the unique affine line of $\mathcal{B}$. The other elements of $\mathcal{B}$ are the projections of $E \backslash D$, with $E$ ranging over all conics of $\mathcal{V}_{2}^{4}$ distinct from $C$ and $D$.

In particular, $d \leq 5$ and $\mathcal{A}$ is always isomorphic to $\operatorname{AG}(2, q)$.

For all the above models, except possibly for $(c 2),(c 3)$ and $(c 4)$, it is clear that Condition (iii) is satisfied. For $(c 3)$ and $(c 4)$, we will show (iii) in our proof; for $(c 2)$, we only have to show that, for any conic $D \neq C$ of $\mathcal{V}_{2}^{4}$, the 3 -space $\Sigma$ generated by $p$ and $D$ does not contain any point $y$ of $\mathcal{V}_{2}^{4} \backslash(D \cup C)$. Choosing two points $z, u \in C \backslash D$ such that $p, z, u$ are on a line of PG $(4, q)$, we see that the conics through $z$ and $y$, and through $u$ and $y$, are contained in the 4-space spanned by $\Sigma$ and $C$, contradicting the fact that every three conics mutually intersecting in three distinct points span the whole space $\mathrm{PG}(5, q)$.

Remark 1.1 For each of these models the set $\mathcal{P}$ is contained in some affine space $\mathrm{AG}(d, q) \subseteq \mathrm{PG}(d, q)$.

We mention the following special cases of the Main Result. Let $\mathcal{A}=(\mathcal{P}, \mathcal{B})$ be as in the Main Result-Affine Planes.

1. If $\mathcal{B}$ does not contain affine lines, then $\mathcal{A}$ is as in $(a),(b 1),(c 2)$ or $(c 3)$.

2. If every plane $q$-arc of $\mathcal{B}$ is contained in a conic, then $\mathcal{A}$ is one of $(a),(b 1)$ where $\mathcal{O}$ is an elliptic quadric, (b2) where the oval $O$ is a conic, $(b 3),(b 4)$ where the oval $O$ is a conic, $(c 1),(c 2),(c 3)$ with $\sigma=2$, or $(c 4)$ with $\sigma=2$. 
3. If $\mathcal{B}$ does not contain affine lines and if every plane $q$-arc of $\mathcal{B}$ is contained in a conic, then $\mathcal{A}$ is one of $(a),(b 1)$ where $\mathcal{O}$ is an elliptic quadric, $(c 2)$, or $(c 3)$ with $\sigma=2$.

Another corollary concerns representations of projective planes.

Main Result-Projective Planes. Let $\Pi=(\mathcal{P}, \mathcal{B})$ be a projective plane of order $q$ with point set $\mathcal{P}$ and set of blocks $\mathcal{B} \subseteq 2^{\mathcal{P}}$. Assume that

(i) the set $\mathcal{P}$ is a point set of $\mathrm{PG}(d, q), d \geq 3$ and $q \geq 7$, with $\langle\mathcal{P}\rangle=\mathrm{PG}(d, q)$;

(ii) the elements of $\mathcal{B}$ are either lines or ovals of $\mathrm{PG}(d, q)$;

(iii) if $L \in \mathcal{B}$ is an oval, then $\langle L\rangle \cap \mathcal{P}=L$.

Then exactly one of the following possibilities occurs.

$(A) d=5$. Here, the set $\mathcal{P}$ is the Veronesean surface $\mathcal{V}_{2}^{4}$ and the elements of $\mathcal{B}$ are the conics on $\mathcal{V}_{2}^{4}$.

$(B) d=3$ and $q$ is even. The set $\mathcal{P}$ is a cone $\mathcal{K}$ with vertex $p$ and base an oval $O$. The blocks are the ovals on $\mathcal{K}$ which are intersections of $\mathcal{K}$ with planes through the nucleus of $O$ and not passing through $p$, together with the generators of $\mathcal{K}$.

$(C) d=4$ and $q$ is even. The coordinates can be chosen such that the set $\mathcal{P}$ consists of all points with coordinates $\left(x_{0}, x_{1}, x_{2}, x_{3}, x_{4}\right)$, where

$$
\left\{\begin{array}{l}
x_{0}=a^{\sigma}, \\
x_{1}=b^{\sigma}, \\
x_{2}=c^{\sigma}, \\
x_{3}=a c^{\sigma-1}, \\
x_{4}=b c^{\sigma-1},
\end{array}\right.
$$

with $a, b, c \in \mathrm{GF}(q),(a, b, c) \neq(0,0,0)$, and $\sigma$ an automorphism which generates $\operatorname{AutGF}(q)$. The set $\mathcal{B}$ contains exactly one line, which has equations $X_{2}=X_{3}=$ $X_{4}=0$ and which corresponds to $c=0$. The other members of $\mathcal{B}$ are translation ovals and coincide with the ovals lying on $\mathcal{P}$.

For $\sigma=2$, the projective plane $\Pi$ can also be described as follows. Let $\mathcal{V}_{2}^{4}$ be the Veronesean surface in $\mathrm{PG}(5, q), q$ even, and let $\mathcal{N}$ be the nucleus plane of $\mathcal{V}_{2}^{4}$. Let $p \in \mathcal{N}$ and let $\mathrm{PG}(4, q)$ be a hyperplane of $\mathrm{PG}(5, q)$ not containing $p$. Then $\mathcal{P}$ is the projection of $\mathcal{V}_{2}^{4}$ from $p$ into $\mathrm{PG}(4, q)$. If $C$ is the unique conic on $\mathcal{V}_{2}^{4}$ with nucleus $p$, then the projection of $C$ is the unique line of $\mathcal{B}$. The other elements of $\mathcal{B}$ are the projections of $D$, with $D$ ranging over all conics of $\mathcal{V}_{2}^{4}$ distinct from $C$. 
As for the case of affine planes, we mention the following special cases. Let $\Pi=(\mathcal{P}, \mathcal{B})$ be as in the Main Result-Projective Planes.

1. If $\mathcal{B}$ does not contain lines, then $\Pi$ is as in $(A)$.

2. If every oval of $\mathcal{B}$ is a conic, then $\Pi$ is one of $(A),(B)$ where $O$ is a conic, or $(C)$ with $\sigma=2$.

We now explain the motivation of our research, and mention an immediate application afterwards, but not related to the original motivation.

Our result embeds in the theory of translation generalized quadrangles of order $\left(q^{n}, q^{2 n}\right)$, for some prime power $q$ and some natural number $n$. This is equivalent with the theory of generalized ovoids via a construction similar to the André-Bose-Bruck construction linking translation planes to certain spreads of projective spaces. For details on translation generalized quadrangles we refer to the monograph of Thas, Thas and Van Maldeghem [5].

A generalized ovoid $O=O(n, 2 n, q)$ consists of a set of $q^{2 n}+1$ subspaces of dimension $n-1$ of PG $(4 n-1, q)$ such that every three such subspaces generate a subspace of dimension $3 n-$ 1 , and such that for every element $\pi$ of $O$, there is a unique subspace $\tau$ of dimension $3 n-1$ which does not meet any other member of $O$. Assume that the generalized quadrangle $\mathcal{S}$ defining $O$ contains a regular line $M$ not containing the point $(\infty)$ of $\mathcal{S}$ and let $M$ be concurrent with the line $\pi \in O$ of $\mathcal{S}$. An arbitrary $3 n$-space $\mathrm{PG}(3 n, q)$ containing $\tau$ intersects the union of all elements of $O$ except $\pi$ in a set $\mathcal{P}$ of $q^{2 n}$ points. One can show that every pair of distinct elements of $\mathcal{P}$ is contained in a conic contained in $\mathcal{P} \cup \pi$ (and each such conic meets $\pi$ in a unique point). If $\mathcal{B}$ is the family of all $q$-arcs obtained by deleting from the previous conics the points belonging to $\pi$, then $(\mathcal{P}, \mathcal{B})$ is an affine space, and every plane of this space is embedded as in our Main Result-Affine Planes. More exactly, by the third special case, there are only four possibilities. This should be a major step in the investigation of such generalized ovoids, more in particular, to prove the conjecture that $O$ is good at $\pi$. An immediate consequence would be a purely geometric proof of the classification of finite Moufang quadrangles (this is the missing part in the monograph of Payne and Thas [4]).

But our main results are also of independent interest. Indeed, up to now it was not known whether all embeddings of finite affine planes in projective 4-space $\mathrm{PG}(4, q)$, where lines are represented by plane $q$-arcs, are covered by model $(a)$. Example $(c 3)$ shows that this is not the case if $q$ is even. For $q$ odd, it is true for those models in PG $(4, q)$ that 
additionally satisfy Assumption ( iii) of our Main Result-Affine Planes. Also, it was not known whether the examples in (b2), with $q$ even and $O$ not a conic, admit coverings. Our theorem shows that they indeed admit a covering as soon as the base plane $q$-arc is a translation oval where the common point of the oval and the axis is removed, see Case $(c 3)$.

\section{Proof of the Main Results}

Throughout this section, we let $\mathcal{A}=(\mathcal{P}, \mathcal{B})$ be an affine plane of order $q$, with point set $\mathcal{P}$ spanning $\mathrm{PG}(d, q)$ and such that all members of $\mathcal{B}$ are either affine lines or plane $q$-arcs of $\mathrm{PG}(d, q)$.

We will denote the subspace of PG $(d, q)$ spanned by sets $S_{1}, S_{2}, \ldots$ of points by $\left\langle S_{1}, S_{2}, \ldots\right\rangle$. In this section we also assume Condition (iii), i.e., for each $q$-arc $L \in \mathcal{B}$ we have $\langle L\rangle \cap \mathcal{P}=$ $L$. Before we start the case distinction with respect to the dimension $d$, we prove a general lemma.

Lemma 2.1 If the block $L \in \mathcal{B}$ is an affine line of $\mathrm{PG}(d, q)$, then $\langle L\rangle \cap \mathcal{P}=L$.

Proof. Let $\langle L\rangle \backslash L=\{x\}$ and assume, by way of contradiction, that $x \in \mathcal{P}$. Consider a block $M$ through $x$ not parallel to $L$. Then $M$ is necessarily a plane $q$-arc. But this contradicts Condition (iii).

\section{$2.1 \quad$ Case $d \geq 5$}

We assume that $q \geq 7$.

Let $L_{1}, L_{2}, L_{3}$ be three blocks of $\mathcal{A}$ pairwise intersecting in a distinct point. Let $P_{1}, P_{2}, P_{3}$ be these three distinct points. It is clear that every point of $\mathcal{A}$, distinct from $P_{1}, P_{2}, P_{3}$, is contained in a block that intersects $L_{1} \cup L_{2} \cup L_{3}$ in three distinct points. Hence PG $(d, q)$ is generated by $L_{1} \cup L_{2} \cup L_{3}$. If $L_{1}$ is an affine line, then $d \leq 4$, a contradiction. Since $d \geq 5$, none of $L_{1}, L_{2}, L_{3}$ is an affine line. In particualr, since $\left\langle L_{1}, L_{2}, L_{3}\right\rangle=\mathrm{PG}(d, q)$, it follows that $d=5$. Hence all blocks of $\mathcal{A}$ are plane $q$-arcs. Note also that with a similar argument, every triple of distinct blocks generates $\mathrm{PG}(5, q)$.

Now let $x$ and $y$ be distinct points of $\mathcal{A}$, and let $L \in \mathcal{B}$ be the unique block containing $x, y$. Let $M_{1}, \ldots, M_{q}$ be the blocks of $\mathcal{A}$ containing $x$ but not $y$, and let $N_{1}, \ldots, N_{q}$ be 
the blocks of $\mathcal{A}$ containing $y$ but not $x$. We may choose this notation in such a way that $M_{i}$ and $N_{i}$ are parallel blocks in $\mathcal{A}$, for all $i \in\{1,2, \ldots, q\}$. Let PG $(3, q)$ be a 3 -space of $\mathrm{PG}(5, q)$ skew to $\langle x, y\rangle$, and project $\mathcal{P} \backslash\{x, y\}$ from $\langle x, y\rangle$ onto $\mathrm{PG}(3, q)$. The projections of all points of $L \backslash\{x, y\}$ coincide with some point $\ell$; the projection of the set $M_{i} \backslash\{x\}$ is some set $M_{i}^{\prime}, i \in\{1,2, \ldots, q\}$, and the projection of $N_{i} \backslash\{y\}$ is denoted by $N_{i}^{\prime}, i \in\{1,2, \ldots, q\}$. As $\mathcal{P}$ generates $\mathrm{PG}(5, q)$, our previous paragraph implies that every pair of blocks of $\mathcal{A}$ generates a 4 -space of $\mathrm{PG}(5, q)$, and so the projection of $\mathcal{P} \backslash L$ is injective. Also, the lines $\left\langle M_{1}^{\prime}\right\rangle,\left\langle M_{2}^{\prime}\right\rangle, \ldots,\left\langle M_{q}^{\prime}\right\rangle$ are pairwise skew, and so are the lines $\left\langle N_{1}^{\prime}\right\rangle,\left\langle N_{2}^{\prime}\right\rangle, \ldots,\left\langle N_{q}^{\prime}\right\rangle$. Also, the point $\ell$ does not belong to $\left\langle M_{1}^{\prime}\right\rangle, \ldots,\left\langle M_{q}^{\prime}\right\rangle,\left\langle N_{1}^{\prime}\right\rangle, \ldots,\left\langle N_{q}^{\prime}\right\rangle$.

Let $\mathcal{H}$ be the hyperbolic quadric containing the generators $\left\langle M_{q-2}^{\prime}\right\rangle,\left\langle M_{q-1}^{\prime}\right\rangle,\left\langle M_{q}^{\prime}\right\rangle$ and $\left\langle N_{1}^{\prime}\right\rangle,\left\langle N_{2}^{\prime}\right\rangle,\left\langle N_{3}^{\prime}\right\rangle$. Then $\left\langle M_{4}^{\prime}\right\rangle$ and $\left\langle N_{4}^{\prime}\right\rangle$ are also generators of $\mathcal{H}$ (as these lines intersect three mutually skew generators; remember $q \geq 7$ ), and by the same token now every line $\left\langle M_{i}^{\prime}\right\rangle$ and $\left\langle N_{i}^{\prime}\right\rangle$ is a generator of $\mathcal{H}, i \in\{1,2, \ldots, q\}$. It is clear that the $\left\langle M_{i}^{\prime}\right\rangle$ belong to one system of generators, say $\mathcal{M}$, and the $\left\langle N_{i}^{\prime}\right\rangle$ to the other system, say $\mathcal{N}$, $i \in\{1,2 \ldots, q\}$. Put $\left\{u_{i}\right\}=\left\langle M_{i}^{\prime}\right\rangle \cap\left\langle N_{i}^{\prime}\right\rangle, i=1,2 \ldots, q$. The point of $\left\langle M_{i}^{\prime}\right\rangle$ not contained in $\left\langle N_{1}^{\prime}\right\rangle \cup\left\langle N_{2}^{\prime}\right\rangle \cup \cdots \cup\left\langle N_{q}^{\prime}\right\rangle$ is denoted by $v_{i}$ and the point of $\left\langle N_{i}^{\prime}\right\rangle$ not on $\left\langle M_{1}^{\prime}\right\rangle \cup\left\langle M_{2}^{\prime}\right\rangle \cup \cdots \cup\left\langle M_{q}^{\prime}\right\rangle$ is denoted by $w_{i}, i=1,2, \ldots, q$. The points $v_{1}, \ldots, v_{q}$ belong to a common generator $V \in \mathcal{N}$ of $\mathcal{H}$ and the points $w_{1}, \ldots, w_{q}$ belong to a common generator $W \in \mathcal{M}$. Let $\tilde{\ell}$ be the intersection of $V$ and $W$.

Let $U \neq L$ be a block of $\mathcal{A}$ parallel to $L$. The projection $U^{\prime}$ of $U$ belongs to $\mathcal{H}$, so is contained in a conic. By injectivity of the projection, $U$ belongs to a conic. Varying $L$, it follows that every block of $\mathcal{A}$ belongs to a conic. Now let $D$ be a block of $\mathcal{A}$ not parallel to $L$ and containing neither $x$ nor $y$. The projection $D^{\prime}$ of $D$ contains at least $q-1 \geq 6$ points of $\mathcal{H}$, contains $\ell$, and is contained in a conic $\widetilde{D}$. Hence $\widetilde{D} \subseteq \mathcal{H}$ and so $\ell \in \mathcal{H}$. It follows that $\widetilde{\ell}=\ell$. Assume without loss of generality that $D$ and $M_{1}$ are parallel in $\mathcal{A}$. Then the intersection $\widetilde{D} \cap M_{1}^{\prime}$ must be $u_{1}$ and so $\widetilde{D}=D^{\prime} \cup\left\{u_{1}\right\}$. Consequently, the projections of all blocks of $\mathcal{A}$ parallel to $M_{i}$ and $N_{i}$, but distinct from $M_{i}$ and $N_{i}$, extend to conics on $\mathcal{H}$ by adding the point $u_{i}, i=1,2, \ldots, q$; the projections of all blocks parallel to $L$, but distinct from $L$, extend to conics on $\mathcal{H}$ by adding the point $\ell$.

Let $D$ and $E$ be parallel to $M_{1}, N_{1}$, with $\left|\left\{D, E, N_{1}, M_{1}\right\}\right|=4$. The conics which extend $D$ and $E$ are denoted by $\bar{D}$ and $\bar{E}$, respectively; let $\bar{D}=D \cup\{d\}$ and $\bar{E}=E \cup\{e\}$. Clearly $d$ and $e$ are projected into $u_{1}$. Assume, by way of contradiction, that $d \neq e$. Then the line $\langle d, e\rangle$ intersects the line $\langle x, y\rangle$. Varying $x, y$ in $L \backslash(D \cup E)$, we see that $\langle d, e\rangle$ must intersect at least three non-concurrent lines of $\langle L\rangle$, implying that $\langle d, e\rangle \subseteq\langle L\rangle$. Hence $\langle L\rangle$ and $\langle D\rangle$ have a line in common, and so do $\langle L\rangle$ and $\langle E\rangle$. Consequently $\langle L, D, E\rangle$ is at most 4-dimensional, a contradiction. Hence $d=e$. We conclude that, if $\left\{C_{1}, C_{2}, \ldots, C_{q}\right\}$ is a parallel class of blocks in $\mathcal{A}$, then there is a unique point $c \in \mathrm{PG}(5, q)$ extending all 
the $q$-arcs $C_{1}, C_{2}, \ldots, C_{q}$ to conics $\bar{C}_{1}, \bar{C}_{2}, \ldots, \bar{C}_{q}$.

Let us denote the points extending the $q$-arcs of $\mathcal{B}$ to conics by $\bar{u}_{1}, \bar{u}_{2}, \ldots, \bar{u}_{q}, \bar{u}_{q+1}$, subscripts chosen in such a way that their projections from $\langle x, y\rangle$ onto $\mathrm{PG}(3, q)$ are the points $u_{1}, u_{2}, \ldots, u_{q}, \ell$, respectively. Consider the $q$ blocks of $\mathcal{A}$ parallel to $M_{1}$. With these $q$-arcs correspond in $\mathrm{PG}(3, q)$ the lines $\left\langle M_{1}^{\prime}\right\rangle,\left\langle N_{1}^{\prime}\right\rangle$ and $q-2$ non-singular conics on $\mathcal{H}$; the latter all contain the points $\ell$ and $u_{1}$. Now the union of the $q$ planes of $\operatorname{PG}(3, q)$ through $\ell$ and $u_{1}$ corresponding to these $q-2$ nonsingular conics, and to the singular conics $M_{1}^{\prime} \cup V$ and $N_{1}^{\prime} \cup W$ contains all points of $\mathcal{H}$ except for $u_{2}, u_{3}, \ldots, u_{q}$. Hence the $(q+1)$ th plane through $\ell$ and $u_{1}$ contains $u_{2}, u_{3}, \ldots, u_{q}$ and so $C^{\prime}=\left\{\ell, u_{1}, u_{2}, \ldots, u_{q}\right\}$ is a nonsingular conic, and it is the projection of $C=\left\{\bar{u}_{1}, \bar{u}_{2}, \ldots, \bar{u}_{q+1}\right\}$. Hence $C$ is contained in the 4-space $\xi_{x, y}=\left\langle L, C^{\prime}\right\rangle$. We choose a point $z \in \mathcal{P}, z \notin L$, and we interchange the roles of $y$ and $z$ to see that $C$ is similarly contained in another 4-space $\xi_{x, z}$, and similarly also in $\xi_{y, z}$. Suppose two of these 4-spaces coincide, say $\xi_{x, y}=\xi_{x, z}$. Then an arbitrary block $T$ not through $x$ and parallel neither to the block $L$ nor to the block $R$ containing $x$ and $z$, has two points in common with the blocks $L$ and $R$; furthermore, the conic corresponding to $T$ also intersects $C$ in a point. Hence $\mathcal{P} \subseteq \xi_{x, y}$, a contradiction. If $\xi_{x, y} \cap \xi_{x, z}=\xi_{x, z} \cap \xi_{y, z}=\xi_{y, z} \cap \xi_{x, y}$, then $x, y, z$ and $C$ are contained in a common 3-space $\Sigma$. Then $\Sigma$ contains the conics corresponding to the blocks joining $x$ and $y$, joining $x$ and $z$, and joining $y$ and $z$, a contradiction. We conclude that $\xi_{x, y} \cap \xi_{y, z} \cap \xi_{x, z}$ is a plane and so $C$ is a planar set. Since it projects on a conic $C^{\prime}$, it is itself a conic. Now we add $C$ to $\mathcal{P}$ and obtain an embedding of the projective closure of $\mathcal{A}$ in $\mathrm{PG}(5, q)$ where lines are conics. By Thas and Van Maldeghem [6], $\mathcal{P} \cup C$ is projectively equivalent to the Veronese surface $\mathcal{V}_{2}^{4}$. This yields part $(a)$ of the Main Result-Affine Planes, and (A) of the Main Result-Projective Planes.

\subsection{Case $d=4$}

We continue to assume $q \geq 7$.

\subsubsection{Generalities}

We first establish the possibilities of configurations of the affine lines in $\mathcal{B}$. If there is no affine line, then this configuration is empty. Hence we may suppose that there is at least one affine line, say $L$. If there was a second affine line $L^{\prime}$ not parallel to $L$ as a block of $\mathcal{A}$, then we consider a third block $K$ intersecting both $L$ and $L^{\prime}$, but not in $L \cap L^{\prime}$. It is clear that, since $q \geq 4$, all points of $\mathcal{A}$ are contained in $\left\langle L, L^{\prime}, K\right\rangle$, which is at most 
3-dimensional, a contradiction. Hence every block which is an affine line is parallel to $L$. A similar argument also implies that, if $L^{\prime}$ is a block which is parallel to $L$, and if $L^{\prime}$ is an affine line, then $\left\langle L, L^{\prime}\right\rangle$ is 3-dimensional.

For each block $K$ not parallel to $L$ there is a unique point $m_{K}$ extending $K$ to an oval, if $q$ is odd, and there are two points $m_{K}$ and $n_{K}$ extending $K$ to a hyperoval, if $q$ is even. We choose a plane $\pi$ in $\mathrm{PG}(4, q)$ skew to $\langle L\rangle$ and we project $\mathcal{P} \backslash L$ from $\langle L\rangle$ into $\pi$. The points of each block $K \in \mathcal{B} \backslash\{L\}$ which has nontrivial intersection $x_{K}$ with $L$ are projected into a line $L_{K}$ minus two points $a_{K}, b_{K}$ in $\pi$. We choose the notation in such a way that, for $q$ odd, $b_{K}$ is the projection of the tangent line at $x_{K}$ to the completion $K \cup\left\{m_{K}\right\}$ of $K$, and $a_{K}$ is the projection of $m_{K}$; for $q$ even we assume that $a_{K}$ corresponds to $m_{K}$ and $b_{K}$ to $n_{K}$. Denote the set of points of $\mathcal{A}$ not on $L$ by $A$.

Suppose now, by way of contradiction, that some point $x \in A \backslash K$ is projected into $L_{K}$. Then all points of $A$ except possibly those of the blocks of $\mathcal{A}$ through $x$ parallel to $L$ or to $K$, or those on the block joining $x$ and $L \cap K$, are contained in the inverse image of $L_{K}$ under the projection. Since there are at least two lines through $x$ completely contained in the 3 -space $\left\langle L, L_{K}\right\rangle$, it is now easy to see that this forces all points of $\mathcal{A}$ to be contained in $\left\langle L, L_{K}\right\rangle$, a contradiction.

Hence, we deduce that the projection is injective, and that no image point coincides with a point $a_{K}$ or $b_{K}$, for any $K \in \mathcal{B} \backslash\{L\}$.

Consider the $q$ blocks distinct from $L$ through some point $p$ of $L$. The projective lines corresponding with their projections into $\pi$ meet in points of type $a_{K}$ and $b_{K}$. It is easy to see that $q \geq 4$ forces these projective lines to meet in a common point; denote it by $x_{p}$. The same thing holds for the $q$ blocks of a fixed parallel class $C$ not containing $L$, and we denote the corresponding point by $x_{C}$. It follows easily that the points $x_{t}$, for $t$ varying in $L$, all lie on the unique line $T$ through $x_{p}$ which is not the image of one of the $q$ aforementioned blocks through $p$ (indeed, $T$ must contain $x_{r}$, for $r$ a point of $L, r \neq p$ since any block through $r$ and $p$, respectively, different from $L$ must meet $q-1$ blocks through $p$ and $r$, respectively, distinct from $L$ ). Since there are exactly $q$ points like this, there remains one point on that line, and we denote it by $x_{\infty}$. A similar argument implies that the $q$ points $x_{C}$ corresponding to the $q$ parallel classes not containing $L$ are contained in a second line through $x_{\infty}$. The projections of the blocks contained in the parallel class of $L$ are the equivalence classes of points with respect to the relation of not lying on the projection of a block intersecting $L$. But clearly, these equivalence classes correspond to the lines in $\pi$ through $x_{\infty}$ distinct from the two particular lines containing the points $x_{p}$ and $x_{C}$. Hence the blocks parallel to $L$ which are $q$-arcs are all completed to an oval by adding the point $\ell=\langle L\rangle \backslash L$. 
Hence, if there is a second block $L^{\prime}$ which is an affine line (necessarily parallel to $L$ ), then, if $\ell^{\prime}=\left\langle L^{\prime}\right\rangle \backslash L^{\prime}$, every block parallel to $L$ and which is a $q$-arc, is completed to a hyperoval by adding $\ell$ and $\ell^{\prime}$ to it. It now follows easily that either there is a unique affine line in $\mathcal{B}$, or there are precisely two (parallel) affine lines in $\mathcal{B}$ (and then $q$ is even), or all blocks of a certain parallel class are affine lines. We treat these three cases separately. Afterwards, we also treat the case where there are no affine lines, of course.

\subsubsection{Precisely one block of $\mathcal{A}$ is an affine line of $\mathrm{PG}(4, q)$}

We continue with the above notation.

The tangent lines at $\ell$ to the ovals arising from the $q$-arcs parallel to $L$ by adding $\ell$ are contained in the common plane $\left\langle L, x_{\infty}\right\rangle$. We claim that these tangent lines coincide. Indeed, consider the projection of $\mathcal{A} \backslash J$ from $\langle J\rangle$ onto some line $Z$ of $\mathrm{PG}(4, q)$, where $J$ is a block parallel to but distinct from $L$ and $Z$ is disjoint from $\langle J\rangle$. Let $K$ be a block not parallel to $L$. Since the above projection from $L$ is injective, the blocks $L, K, J$ generate $\mathrm{PG}(4, q)$, and since $J$ and $K$ meet in a point, the projection from $\langle J\rangle$ is injective on the set $K \backslash J$. Hence, if two points have the same projection, then they belong to a block parallel to $L$. Let $J^{\prime}$ be a second block parallel to $L, J^{\prime} \neq J$. If $\left\langle J, J^{\prime}\right\rangle$ is 4-dimensional, then the projection from $\langle J\rangle$ is injective on the set $J^{\prime} \backslash J$. Since $q \geq 4$, we find at least two points $z_{1}, z_{2}$ in $K$ and two points $y_{1}, y_{2}$ in $J^{\prime}$ such that the projection from $\langle J\rangle$ of $z_{i}$ coincides with that of $y_{i}, i \in\{1,2\}$. For at most one $i \in\{1,2\}$, we have $y_{i}=z_{i}$. Suppose $y_{1} \neq z_{1}$; it follows from the previous that $y_{1}$ and $z_{1}$ are contained in a block parallel to $L$, hence $z_{1} \in J^{\prime}$, a contradiction. Hence $\left\langle J, J^{\prime}\right\rangle$ is 3-dimensional. If $\langle J\rangle \cap\left\langle J^{\prime}\right\rangle$ is not tangent to $J \cup\{\ell\}$ at $\ell$, then $\left\langle J^{\prime}\right\rangle$ contains some point of $J$, a contradiction. This shows our claim. We denote the common tangent line at $\ell$ to all ovals corresponding with blocks parallel to $L$ by $T_{\ell}$.

Now we forget about the previous projections and we project from $\ell$ onto a solid $\Sigma$, where $\ell \notin \Sigma$. Then the previous properties of the blocks parallel to $L$ imply easily that the projection of $\mathcal{A}$ is contained in a cone $\mathcal{C}$ with vertex the projection $x_{T}$ of $T_{\ell}$ and base a plane $q$-arc. But the projections of all the blocks not parallel to $L$ go through the common point $x_{L}$, which is the projection of $L$ in $\Sigma$. Let $M$ be the line of $\Sigma$ through $x_{T}$ extending the cone to a cone $\mathcal{C}^{\prime}$ over an oval, if $q$ is odd, and let $M$ and $N$ be the two lines of $\Sigma$ through $x_{T}$ extending the cone to a cone $\mathcal{C}^{\prime}$ over a hyperoval, if $q$ is even.

If $q$ is odd, the line tangent at $x \in L$ to any block $K$ intersecting $L$ in $x$ is projected onto a line contained in the plane tangent to $\mathcal{C}^{\prime}$ at the line $\left\langle x_{T}, x_{L}\right\rangle$. Hence all such tangents are contained in a fixed 3 -space containing $L$, and are hence projected from $L$ onto a line 
of $\pi$. That line contains whence all points $b_{K}$; it also contains the point $x_{\infty}$. It follows that either all pencils with vertex on $L$ correspond with points of type $b_{K}$, or all parallel classes do.

Next we want to show that, still for $q$ odd, all pencils with vertex on $L$ correspond with points of type $b_{K}$. So suppose by way of contradiction that parallel classes correspond with points of type $b_{K}$. Consider two arbitrary blocks $K_{1}, K_{2}$ such that $\left\langle K_{1}\right\rangle,\left\langle K_{2}\right\rangle$ meet $\langle L\rangle$ in different points, and suppose that $\left\langle K_{1}, K_{2}\right\rangle$ is 3-dimensional. Our assumption implies that $\langle L\rangle$ belongs to $\left\langle K_{1}, K_{2}\right\rangle$, contradicting the injectivity of the projection from $\langle L\rangle$.

Now we use a fourth projection. Let $K$ be an arbitrary block not parallel to $L$. Let $\lambda$ be a line of $\mathrm{PG}(4, q)$ skew to $\langle K\rangle$. Then we project the point set $\mathcal{P} \backslash K$ from $\langle K\rangle$ onto $\lambda$. It is clear that $\mathcal{P} \cap\langle K, L\rangle=K \cup L$. Hence $L \backslash K$ gets projected onto a unique point $p_{L}$ of $\lambda$, and all other points of $\mathcal{P} \backslash K$ are projected onto points of $\lambda$ different from $p_{L}$. Suppose two points $x, y \in \mathcal{P} \backslash(L \cup K)$ are projected onto the same point $a$. Then the block $K^{\prime}$ through $x, y$ contains two points in a 3 -space $\Sigma^{\prime}$ containing $K$. If $K^{\prime}$ is not parallel to $K$, then also $K \cap K^{\prime} \in \Sigma^{\prime}$ and hence all points of $K^{\prime}$ belong to $\Sigma^{\prime}$. The previous paragraph implies that $K \cap K^{\prime} \in L$. If $K^{\prime}$ is parallel to $K$, then $\left\langle K, K^{\prime}\right\rangle$ is 4-dimensional, and so no further point of $K^{\prime}$ is projected onto $a$. Now suppose some further point $z$ of $\mathcal{P} \backslash\left(L \cup K \cup K^{\prime}\right)$ is mapped onto $a$. Then the blocks through $x, z$ and through $y, z$ are not parallel to $K$ and belong to $\Sigma^{\prime}$ and so should meet on $L$, contradicting the choice of $z$.

So we see that each point of $\lambda$ is the image of either $0,1,2$ or $q-1$ points of $\mathcal{A} \backslash K$. So we have to divide $q(q-1)$ points in $q+1$ sets of size $0,1,2$ and $q-1, q \geq 5$. If we have $k$ sets of size $q-1$, then $k(q-1)+2(q+1-k) \geq q^{2}-q$, implying $k \geq \frac{q^{2}-3 q-2}{q-3}=q-\frac{2}{q-3}$. Hence, since $q \geq 7$, we infer $k=q$ and we can conclude that all blocks meeting $L$ in $K \cap L$ lie in common 3-spaces with $K$. But, more importantly, the projection is injective on the set of points of any block parallel to $K$ ! This injectivity and the reasoning leading to it also hold for $q$ even. This means that, given such a block $K^{\prime}$, putting $x_{K}=K \cap L, x_{K^{\prime}}=K^{\prime} \cap L$, and $t$ being the intersection of the tangent lines to $K \cup\left\{m_{K}\right\}$ and $K^{\prime} \cup\left\{m_{K^{\prime}}\right\}$ at $x_{K}$ and $x_{K^{\prime}}$, respectively $(t$ exists by the assumption that parallel classes correspond with points of type $b_{K}$ ), the projection of $K^{\prime}$ from $t$ onto any line of $\left\langle K^{\prime}\right\rangle$ not through $t$ is injective, implying that $q$ is even. Now let $q=5$. Then either the above holds, and we have a contradiction, or $k=q-1=4$ and the other two points on $\lambda$ are images of two pairs of points, necessarily pairs on blocks parallel to $K$. But this is a contradiction, as these four remaining points of course lie on the remaining block through the intersection point $K \cap L$.

So we have shown that, if $q$ is odd, then the projection from $\langle L\rangle$ onto $\pi$ of every block except for $L$ of a pencil of blocks containing the block $K \neq L$ and with vertex on $L$, is 
contained in a line that contains $b_{K}$. If $q$ is even, then we can choose the notation of $N$ and $M$ such that the points $b_{K}$ correspond with the line $N$, and the points $a_{K}$ with $M$. Remember that in both cases, with each point $a_{K}$ corresponds the point $m_{K}$ in $\langle K\rangle$ extending $K$ to an oval. We now claim that, since $q \geq 5$, we have $m_{K}=m_{K^{\prime}}$ as soon as $K$ and $K^{\prime}$ are two parallel blocks not parallel to $L$. Indeed, suppose by way of contradiction that $m_{K} \neq m_{K^{\prime}}$. Then $a_{K}=a_{K^{\prime}}$ implies that the line $\gamma$ of PG $(4, q)$ joining $m_{K}$ with $m_{K^{\prime}}$ intersects $\langle L\rangle$ in some point $m$. Hence the projection of $K^{\prime} \cup\left\{m_{K^{\prime}}\right\}$ from $\langle K\rangle$ onto $\lambda$ is injective except that it agrees on $m_{K^{\prime}}$ and $x_{K^{\prime}}$. Hence the projection of $K^{\prime} \cup\left\{m_{K^{\prime}}\right\}$ from $t:=\langle K\rangle \cap\left\langle K^{\prime}\right\rangle$ onto some line in $\left\langle K^{\prime}\right\rangle$ not containing $t$ is injective except for $m_{K^{\prime}}$ and $x_{K^{\prime}}$, whose images agree. But the injectivity implies that $t$ is a nucleus of $K^{\prime} \cup\left\{m_{K^{\prime}}\right\}$, contradicting the fact that there is a unique secant through $t$. So we can add $m_{K}$ to every block $K \neq L$ and obtain a projective plane $\Pi$ when calling the set consisting of $\ell$ and all such $m_{K}$ a block $B$.

The arguments of the previous paragraphs imply that, if $K, K^{\prime}$ are blocks distinct from $L$ with $x_{K}=x_{K^{\prime}}$, then $\left\langle K, K^{\prime}\right\rangle$ is a 3 -space. Since $L$ does not belong to that 3 -space (as we noted earlier), the tangents to $K \cup\left\{m_{K}\right\}$ and $K^{\prime} \cup\left\{m_{K^{\prime}}\right\}$ at $x_{K}$ coincide.

If we project from $x_{K}$ instead of from $\ell$ above, then we see that the projection of $(\mathcal{A} \cup B) \backslash$ $\left\{x_{K}\right\}$ lies on a cone with base an oval. Since $B$ lies in a plane (the projection of $B \backslash\{\ell\}$ from $\ell$ onto $\Sigma$ yields the line $M$ ), it is an oval. Now, if one considers a point $x \in A$, then the tangent lines at $x$ to the ovals through $x$ are contained in a 3 -space containing $\ell$ (since after projection from $\ell$, they are contained in a plane), and in a 3 -space containing $x_{K}$. These 3 -spaces are clearly distinct, and so all these tangent lines are contained in a plane (and they fill up the whole plane).

Now we show that $q$ is even. Assume, by way of contradiction, that $q$ is odd. Let $T_{1}, T_{2}, \ldots, T_{q}, T_{\infty}$ be the $q+1$ tangent lines at the respective points of $L \cup\{\ell\}$ of the $q^{2}+q$ ovals of $\Pi$ (where $\ell \in T_{\infty}$ ). Note that all these tangents are contained in a 3 -space $\Sigma_{T}$. Let $x$ be an arbitrary point of $\Pi$ not on $\langle L\rangle$. The tangent lines at $x$ of the $q+1$ ovals containing $x$ intersect the respective lines $T_{1}, \ldots, T_{q}, T_{\infty}$ in collinear points $t_{1}^{x}, \ldots, t_{q}^{x}, t_{\infty}^{x}$ (all these points are on the line $L_{x}$ which is the intersection of $\Sigma_{T}$ and the plane containing all the aforementioned tangent lines at $x$ ). This way there arise $q$ transversals $L_{x}$ of the lines $T_{1}, T_{2}, \ldots, T_{q}, T_{\infty}$ (vary $x$ on one oval of $\Pi$ and take account of $q$ being odd), and, adding $\langle L\rangle$, we obtain the two systems of generators of a hyperbolic quadric $\mathcal{H}$ in the solid $\Sigma_{T}$. Let $O$ be an oval of $\Pi$ not containing the point $x$ of $\Pi$, with $x \notin L \cup\{\ell\}$. Let $y \in O$, then the line $\langle x, y\rangle$ intersects the tangent $T_{i}$, where $i$ is such that $T_{i}$ is tangent to the oval through $x$ and $y$. It follows that the projection of $O$ from $x$ into $\Sigma_{T}$ is an oval lying completely on $\mathcal{H}$. But if $T_{j}$ contains $O \cap\langle L\rangle$, then $T_{j}$ is clearly tangent to the projection of $O$, a contradiction. Hence $q$ is even. 
Let $O$ be an oval of $\Pi$ and let $x, y$ be two points of $O$ off $\langle L\rangle$. Pick an arbitrary point $z$ of $\Pi$ not in $O \cup\langle L\rangle$. Let the ovals of $\Pi$ through $x$ and $z$, and through $y$ and $z$ have tangent lines $T_{x}$ and $T_{y}$, respectively, at their intersection with $\langle L\rangle$. Let $O^{\prime}$ be the oval of $\Pi$ containing $z$ and $O \cap\langle L\rangle$. Then the projection from $T_{x}$ of $\langle O\rangle$ onto $\left\langle O^{\prime}\right\rangle$, followed by the projection from $T_{y}$ of $\left\langle O^{\prime}\right\rangle$ onto $\langle O\rangle$ maps $O$ onto itself, fixes every point of $\langle O\rangle \cap\left\langle O^{\prime}\right\rangle$ (and this is the tangent line to $O$ at $O \cap\langle L\rangle$ ) and maps $x$ to $y$. Hence $O$ is a translation oval with center $O \cap\langle L\rangle$. By projection from any point of $\langle L\rangle$ it is clear that all ovals in $\Pi$ are isomorphic.

We now show that $\mathcal{P} \cup B$ is projectively unique given the isomorphism class of translation ovals belonging to $\Pi$. We choose a point $p \in L$ and two translation ovals $O_{1}, O_{2}$ of $\Pi$ containing the translation center $p$. These ovals generate a 3 -space $\Sigma$ and have a common tangent line at $p$. Moreover, by the previous paragraph, the tangent line at $\ell$ to the ovals through $\ell$ meets $\Sigma$ in a point $t$ which is the vertex of a cone $\mathcal{C}$ containing $O_{1}, O_{2}$ and such that the generators of that cone define an isomorphism between $O_{1}$ and $O_{2}$. We see that the configuration $O_{1}, O_{2}, \ell, t$ is projectively unique. Also, let $x \in \mathcal{P} \backslash\left(L \cup O_{1} \cup O_{2}\right)$; then $x \notin \Sigma$. We may choose $x$ such that $\langle\ell, x\rangle$ intersects $\mathcal{C}$ at a given point $x^{\prime}$ (distinct from $t$ and off $O_{1} \cup O_{2}$ ). The projections from $\ell$ onto $\Sigma$ of the ovals of $\Pi$ through $x$ but not through $\ell$ are given by the ovals of $\mathcal{C}$ through $x^{\prime}$ and $p$; the projection from $\ell$ onto $\Sigma$ of the oval of $\Pi$ containing $\ell$ and $x$, with $\ell$ removed, is the set $\left\langle x^{\prime}, t\right\rangle \backslash\{t\}$.

Let $O^{\prime}$ be such an oval of $\mathcal{C}$ containing $x^{\prime}$ and $p$, and let $O^{\prime} \cap O_{1}=\left\{x_{1}\right\}$ and $O^{\prime} \cap O_{2}=\left\{x_{2}\right\}$. Then the intersection of the cone with base $O^{\prime}$ and vertex $\ell$ with the plane $\left\langle x_{1}, x_{2}, x\right\rangle$ is an oval of $\Pi$ containing $x$. If $\left\langle x^{\prime}, t\right\rangle \cap O_{1}=\left\{y_{1}\right\}$ and $\left\langle x^{\prime}, t\right\rangle \cap O_{2}=\left\{y_{2}\right\}$, then for the given automorphism of $\mathrm{GF}(q)$ the translation oval with translation line $\langle\ell, t\rangle$ and containing $\ell, y_{1}, y_{2}, x$ is an oval of $\Pi$ containing $x$. It follows that all ovals through $x$ are determined, and hence $\mathcal{P} \cup B$ is projectively unique.

Since a translation oval is determined by its associated field automorphism, it remains to show that Case $(C)$ really defines a projective plane satisfying the conditions $(i),(i i)$ and (iii) of the Main Result-Projective Planes, and that it contains exactly one line of $\operatorname{PG}(4, q)$.

Let $\sigma$ be an automorphism of $\operatorname{GF}(q)$ which generates $\operatorname{AutGF}(q), q$ even, and read $\sigma$ as a natural number $2^{e}$, with $e>0$, so that $x^{\sigma-1}$ is well defined for all $x \in \operatorname{GF}(q)$. Let us consider $\mathrm{PG}(2, q)$ using ordinary homogenous coordinates for the points and lines. We define the following map $\tau$ of the points of $\mathrm{PG}(2, q)$ to a point set of $\mathrm{PG}(4, q)$.

$$
(x, y, z)^{\tau}=\left(x^{\sigma}, y^{\sigma}, z^{\sigma}, x z^{\sigma-1}, y z^{\sigma-1}\right) .
$$


The images of the points $(1,0,0),(0,1,0),(0,0,1),(1,0,1)$ and $(0,1,1)$ clearly generate PG $(4, q)$. Whence $(i)$.

The line $[0,0,1]$ clearly corresponds with the line $X_{2}=X_{3}=X_{4}=0$ (labeling the coordinates in $\mathrm{PG}(4, q)$ by $\left.X_{0}, X_{1}, \ldots, X_{4}\right)$.

By the symmetry of $\tau$ in the first two coordinates we may assume that a generic line $M$ of PG $(2, q)$ has coordinates $[1, b, c]$, i.e., the points $(x, y, z)$ of the line satisfy $x=b y+c z$. Applying $\tau$, we obtain the set

$$
\left\{\left(b^{\sigma} y^{\sigma}+c^{\sigma} z^{\sigma}, y^{\sigma}, z^{\sigma}, b y z^{\sigma-1}+c z^{\sigma}, y z^{\sigma-1}\right): y, z \in \mathrm{GF}(q)\right\} .
$$

We see that this set of points is contained in the plane $\pi$ with equations

$$
\left\{\begin{array}{l}
X_{0}=b^{\sigma} X_{1}+c^{\sigma} X_{2} \\
X_{3}=b X_{4}+c X_{2}
\end{array}\right.
$$

Projecting this set of points from the line spanned by $(1,0,0,0,0)$ and $(0,0,0,1,0)$ into the plane with equations $X_{0}=X_{3}=0$, we obtain the set $\{(0,1,0,0,0)\} \cup\left\{\left(0, y^{\sigma}, 1,0, y\right)\right.$ : $y \in \operatorname{GF}(q)\}$, which is clearly a translation oval with associated field automorphism $\sigma$. This proves $($ ii $)$.

Suppose now that some point $(u, v, w)^{\tau}$ is contained in the plane $\pi$ above. Then it satisfies $X_{0}=b^{\sigma} X_{1}+c^{\sigma} X_{2}$, hence $u^{\sigma}=b^{\sigma} v^{\sigma}+c^{\sigma} w^{\sigma}$, implying $u=b v+c w$, and so $(u, v, w)$ belongs to the line $M$. This shows $(i i i)$.

It remains to show that the only ovals on $\mathrm{PG}(2, q)^{\tau}$ are the images of the lines distinct from $[0,0,1]$. This will follow as soon as we prove that no five points are contained in a common plane not generated by the image of a line $M \neq[0,0,1]$ of $\mathrm{PG}(2, q)$ (note $q>4$ ). Suppose five points $p_{1}, \ldots, p_{5}$ of $\mathrm{PG}(2, q)^{\tau}$ are contained in a common plane $\alpha$, where $\alpha$ is not a plane spanned by the image of a line of $\mathrm{PG}(2, q)$, distinct from $[0,0,1]$. We may suppose that at most one of the points $p_{1}, \ldots, p_{5}$ is contained in the line $[0,0,1]^{\tau}$. Hence the oval $O_{i, j}$ through $p_{i}, p_{j}$, with $O_{i, j}=N^{\tau}$ and $N$ a line of PG $(2, q)$, does not contain any point of $\alpha$ besides $p_{i}, p_{j}, 1 \leq i<j \leq 5$. Let the ovals $O_{1,2}$ and $O_{3,4}$ intersect in a point $p$. Then clearly $O_{1,2}$ and $O_{3,4}$ are contained in the 3 -space $\langle\alpha, p\rangle$. It is now easy to see that, using $(i i)$, all points of $\mathrm{PG}(2, q)^{\tau}$ are contained in that 3-space, contradicting $(i)$.

This completes the case of one affine line, by deleting from the foregoing block $B$ and noting that we may take for $B$ the image of the line $[0,1,0]$ (indeed, one verifies that all choices are projectively equivalent). We obtain Case $(c 4)$ of Main Result-Affine Planes. Adding $B$ again, we obtain Case $(C)$ of Main Result-Projective Planes.

It is also easy to check that, if $\sigma=2$, then in the projective case we obtain the projection of a Veronesean surface $\mathcal{V}_{2}^{4}$ from a point $n$ of its nucleus plane, and in the affine case we delete from $\mathcal{V}_{2}^{4}$ the conic with nucleus $n$ and then project from $n$. 


\subsubsection{Precisely two blocks of $\mathcal{A}$ are affine lines of $\mathrm{PG}(4, q)$}

We have already shown in Paragraph 2.2.1 that in this case $q$ is even. Let $L$ and $L^{\prime}$ be the two affine lines in $\mathcal{B}$, and let $\ell$ and $\ell^{\prime}$ be the points of $\operatorname{PG}(4, q)$ on $\langle L\rangle$ and $\left\langle L^{\prime}\right\rangle$, respectively, but not belonging to $\mathcal{P}$. We have also shown above that $L$ and $L^{\prime}$ are parallel blocks of $\mathcal{A}$.

Let $K$ be a block intersecting $L$ in $x_{K}$ and $L^{\prime}$ in $x_{K}^{\prime}$. We consider the projection of $\mathcal{P} \backslash K$ from $\langle K\rangle$ onto $\lambda:=\left\langle\ell, \ell^{\prime}\right\rangle$ (note that $\lambda$ and $\langle K\rangle$ are indeed skew since otherwise $\mathcal{P}$ is contained in the 3 -space $\langle K, \lambda\rangle)$. Suppose some point $x \in \mathcal{P} \backslash\left(K \cup L \cup L^{\prime}\right)$ is projected onto $\ell$. Then every block through $x$ not parallel to $L$ and not parallel to $K$ is contained in $\langle K, L\rangle$, and hence all points of $\mathcal{A}$ are contained in the 3 -space $\langle K, L\rangle$, a contradiction. Similarly, no point of $\mathcal{P} \backslash\left(K \cup L \cup L^{\prime}\right)$ is projected onto $\ell^{\prime}$. Now suppose three points $x, y, z \in \mathcal{P} \backslash\left(K \cup L \cup L^{\prime}\right)$ are projected onto the same point $p$. The block through $x, y$ is a $q$-arc $K_{x, y}$. By Paragraph 2.2.1 $K_{x, y}$ is not parallel to $L$, as otherwise it contains $\ell$ and $\ell^{\prime}$. Suppose first that $K_{x, y}$ is not parallel to $K$ and hence intersects $K$ in some point $u$. Then $K_{x, y} \subseteq\langle K, p\rangle$. At least one of $L, L^{\prime}$ does not contain $u$, hence is also contained in $\langle K, p\rangle$, and so all points of $\mathcal{A}$ are contained in $\langle K, p\rangle$, a contradiction. Hence $x, y, z$ are contained in a block $K_{x, y}$ parallel to $K$ and again $K_{x, y} \subseteq\langle K, p\rangle$, leading to the same contradiction as above. Hence the number of points of $\mathcal{P} \backslash K$ projected onto $\ell$ and $\ell^{\prime}$ is precisely $q-1$ (for each), and every other point on $\lambda$ is the image of at most two points of $\mathcal{P}$. Hence $q^{2}-q \leq 2(q-1)+2(q-1)$, implying $q^{2} \leq 5 q-4$, or $q \in\{2,4\}$, the final contradiction for this case.

\subsubsection{At least three blocks of $\mathcal{A}$ are affine lines of $\mathrm{PG}(4, q)$}

Here, we know by Paragraph 2.2.1 that all blocks of a parallel class of $\mathcal{A}$ are affine lines, and no other block is an affine line. Let $L_{1}, L_{2}, \ldots, L_{q}$ be the affine lines of $\mathcal{B}$, and put $\ell_{i}=\left\langle L_{i}\right\rangle \backslash L_{i}, i=1,2, \ldots, q$. From Paragraph 2.2.1, we infer that all $\ell_{i}$ are contained in a plane together with $L_{j}$, for every $j \in\{1,2, \ldots, q\}$. Taking intersections of two of these planes, we deduce that all $\ell_{i}$ are contained in a line $L_{\infty}$ of $\operatorname{PG}(4, q)$. Consider a point $x \in L_{1}$ and project $\mathcal{P} \backslash\{x\}$ from $x$ onto some 3 -space $\Sigma \not \ngtr x$. The projections of the blocks through $x$ distinct from $L_{1}$, together with the projection of $L_{\infty}$, and the projections of the $L_{i}, i=2,3, \ldots, q$, are respectively contained in the two systems of generators of a hyperbolic quadric $\mathcal{H}$. Every block $K$ not through $x$ and distinct from $L_{i}, i=2, \ldots, q$, is entirely projected into $\mathcal{H}$, which implies that $K$ is contained in a conic $\widetilde{K}$, which is uniquely determined by $K$ and $\mathcal{H}$ (since $q>4$, we even do not need $\mathcal{H}$ for the uniqueness). Let $\widetilde{k}=\widetilde{K} \backslash K$. Then the projection of the point $\widetilde{k}$ is contained in the unique generator 
not containing the projection of any point of $\mathcal{A} \backslash\{x\}$. Hence all points $\widetilde{k}$ together with $x$ are contained in a plane. This plane does not contain the line $L_{1}$. Considering another choice $x^{\prime} \in L_{1}$ for $x$ we see that for all $q$-arcs $K$ not containing $x$ nor $x^{\prime}$ the points $\widetilde{k}$ are contained in a line $L_{q+1}$. Also introducing the points $\widetilde{k}$ for all $q$-arcs on $x$, it is now clear that for all $q$-arcs the points $\widetilde{k}$ are on the line $L_{q+1}$. Choosing for $x$ a point $x^{\prime \prime}$ on $L_{2}$ we see that $L_{q+1}$ contains a point of $\left\langle x, L_{\infty}\right\rangle \cap\left\langle x^{\prime \prime}, L_{\infty}\right\rangle=L_{\infty}$ which is necessarily the point $L_{\infty} \backslash\left\{\ell_{1}, \ell_{2}, \ldots, \ell_{q}\right\}$.

It is clear that projection from $L_{\infty}$ of any block $K$ onto the plane $\left\langle K^{\prime}\right\rangle$ of any other block $K^{\prime}$, with $K$ and $K^{\prime} q$-arcs, coincides with $K^{\prime}$. Conversely, the projection from $\langle K\rangle$ onto $L_{\infty}$ of the unique conic containing $K^{\prime}$ is bijective. It follows that cross-ratios are preserved and hence $\mathcal{P} \cup L_{\infty} \cup L_{q+1}$ is a rational normal cubic scroll. So we obtain Case $(c 1)$ of Main Result-Affine Planes. Remark that a rational normal cubic scroll can be viewed as the projection of a Veronesean surface $\mathcal{V}_{2}^{4}$ from a point $u \in \mathcal{V}_{2}^{4}$ into a hyperplane $\mathrm{PG}(4, q)$ not containing $u$.

Since the projective lines corresponding with the blocks which are affine lines do not meet in a point, this model cannot be extended to a projective plane.

\subsubsection{All lines of $\mathcal{A}$ are planar $q$-arcs of $\mathrm{PG}(4, q)$}

Let $K$ be any block, and let $L$ be a line of PG $(4, q)$ skew to $\langle K\rangle$. We project $\mathcal{P} \backslash K$ on $L$ from $\langle K\rangle$. Suppose three points $x, y, z \in \mathcal{P} \backslash K$ are projected onto the same point $p \in L$. If $x, y, z$ are contained in a block $D$, then all points of $D \backslash K$ are projected onto $p$ (and there are $q$ or $q-1$ such points). If some additional point $u \in \mathcal{P} \backslash(K \cup D)$ is projected onto $p$, then it is easy to see that $\mathcal{P}$ is contained in the inverse image of $p$, which is a 3 -space, a contradiction. If $x, y, z$ are not contained in a block, then we may suppose that the block $D$ containing $x$ and $y$ is not parallel to $K$, and so $\langle K, D\rangle$ is 3-dimensional. But $z$ is an additional point of $\mathcal{P}$ contained in $\langle K, D\rangle$, a contradiction. Hence it follows that the pre-image of a point on $L$ with respect to the projection is either empty, or contains $1,2, q-1$ or $q$ points. Now note that $q$ and $q-1$ cannot occur both since, if $K$ and an intersecting block $D$ are contained in a 3-space, and also $K$ and a parallel block $D^{\prime}$, then $\left\langle D \cap D^{\prime}, K\right\rangle=\langle D, K\rangle=\left\langle D^{\prime}, K\right\rangle$, a contradiction. Also, if the inverse image contains exactly two points, then these two points belong to a block parallel to $K$.

Suppose first that $q$ does not occur and let $m$ be the number of times $q-1$ occurs. Then $q^{2}-q \leq m(q-1)+2(q+1-m)$, which implies $m \geq \frac{q^{2}-3 q-2}{q-3}$. Since $q \geq 5$, we deduce $m \in\{q, q-1\}$. Since the blocks defined by the $m$ pre-images cannot meet off $K$, we either have $m$ blocks through a point of $K$, or $m$ blocks of a parallel class distinct from the class 
of $K$. If $m=q-1$, then the $q-1 \geq 4$ points remaining lie on a unique block, which is by the foregoing not parallel to $K$, contradicting a previous observation. So in all cases $m=q$, and we see that either there is a point $x_{K}$ on $K$ such that the plane of every block through $x_{K}$ meets $\langle K\rangle$ in at least a line, or there is some parallel class of blocks distinct from the class of $K$ such that the plane of every member of that class intersects $\langle K\rangle$ in a line.

Now suppose that $q-1$ does not occur and let $m$ be the number of times $q$ occurs as size of the pre-image of a point of $L$. Similarly as above we obtain $m \geq q-1-\frac{4}{q-2}$. If $q>6$, then $m=q-1$. Hence, interchanging the roles of $K$ and any parallel block, we see that in this case every pair of blocks of the parallel class of $K$ is contained in a 3-space.

Now assume that $K$ is contained in a 3-space together with every block through some point $x_{K} \in K$. Let $K^{\prime} \in \mathcal{B} \backslash\{K\}$ and let $K$ and $K^{\prime}$ meet in a point $y \neq x_{K}$. Then there is always a block $D \neq K$ through $x_{K}$ contained with $K^{\prime}$ in a 3 -space. Hence $D$ is contained with $K$ in a 3 -space, and also with $K^{\prime}$ in a 3 -space. So $\langle D, K\rangle=\langle D, y\rangle=\left\langle D, K^{\prime}\right\rangle$, a contradiction. Consequently this case does not occur. We have shown that for every block $K$, the blocks contained with $K$ in a 3-space form a unique parallel class of blocks.

This easily implies that there is an involutorial mapping $\iota$ on the family of parallel classes of $\mathcal{A}$ such that every member of a parallel class $\mathfrak{P}$ is contained in a 3 -space together with every member of the parallel class $\mathfrak{P}^{\iota}$. We call $\iota$ a pairing, and we say that a parallel class is self-paired if it is fixed under $\iota$.

We now proceed with a case distinction depending on the number of self-pairings.

\section{There are at least three self-pairings, i.e., $\iota$ has at least three fixed elements}

Note that the planes of the blocks of any self-paired parallel class $P$ contain a common line $L_{P}$ of PG $(4, q)$, as otherwise we can find three parallel blocks contained in a 3 -space, and then $\mathcal{P}$ belongs to that 3 -space, a contradiction.

Let $\mathfrak{P}_{1}, \mathfrak{P}_{2}, \mathfrak{P}_{3}$ be three self-paired parallel classes of blocks, and let $x \in \mathcal{P}$ be arbitrary. Let $K_{i}, i=1,2, \ldots, q+1$, be the blocks containing $x$, and suppose $K_{j} \in \mathfrak{P}_{j}$ for $j \in$ $\{1,2,3\}$. There are precisely two solids $\Sigma_{j}^{(1)}, \Sigma_{j}^{(2)}$ intersecting $\mathcal{P}$ in precisely $K_{j}, j=1,2,3$. Clearly, these solids intersect $\left\langle K_{i}\right\rangle$ (if they do not contain that plane) in a tangent to $K_{i}$, $i=1,2, \ldots, q+1$. Suppose that the solid $\Sigma_{1}^{(n)}$ intersects $\left\langle K_{i}\right\rangle$ in the tangent $T_{i}^{(n)}, n=1,2$, $i=2,3, \ldots, q+1$; as $\Sigma_{1}^{(1)} \neq \Sigma_{1}^{(2)}$ we have $T_{i}^{(1)} \neq T_{i}^{(2)}$. Then all $T_{i}^{(1)}, i=2,3, \ldots, q+1$, are contained in the union of the two distinct planes $\Sigma_{1}^{(1)} \cap \Sigma_{2}^{(1)}$ and $\Sigma_{1}^{(1)} \cap \Sigma_{2}^{(2)}$, which intersect in $T_{2}^{(1)}$, and also in the two distinct planes $\Sigma_{1}^{(1)} \cap \Sigma_{3}^{(1)}$ and $\Sigma_{1}^{(1)} \cap \Sigma_{3}^{(2)}$, which intersect in 
$T_{3}^{(1)} \neq T_{2}^{(1)}$. It follows easily that we may assume that $\Sigma_{1}^{(1)} \cap \Sigma_{2}^{(1)}=\Sigma_{1}^{(1)} \cap \Sigma_{3}^{(1)}=: \alpha$. The number of distinct tangents $T_{i}^{(1)}, i=2,3, \ldots, q+1$, is at least $2+\frac{q-2}{2}=\frac{q}{2}+1$, so $\alpha$ contains at least $\frac{q}{2}$ tangents. Assume by way of contradiction that neither $T_{1}^{(1)}$ nor $T_{1}^{(2)}$ is contained in $\alpha$, where $T_{1}^{(1)}$ and $T_{1}^{(2)}$ are the tangents of $K_{1}$ at $x$. Then $\alpha \cap\left\langle K_{1}\right\rangle$ is a point. So we find at least $\frac{q}{2} 3$-spaces $\left\langle K_{1}, L\right\rangle$, with $L$ a tangent in $\alpha$, intersecting $\mathcal{P}$ in $K_{1}$, clearly a contradiction. Hence we may assume that $T_{1}^{(1)} \subseteq \alpha$. Clearly $T_{1}^{(2)}$ is not contained in $\alpha$. Hence $T_{1}^{(2)}=\Sigma_{1}^{(1)} \cap \Sigma_{2}^{(2)} \cap \Sigma_{3}^{(2)}$. As $T_{i}^{(1)} \neq T_{1}^{(2)}, i=1,2, \ldots, q+1$, we conclude that $T_{1}^{(1)}, T_{2}^{(1)}, \ldots, T_{q+1}^{(1)}$ are contained in $\alpha$. Likewise, there is a plane $\beta$ containing $T_{i}^{(2)}$, for all $i \in\{1,2, \ldots, q+1\}$, and $\alpha \cap \beta=\{x\}$ (the latter also follows from $\left\langle K_{1}, K_{2}\right\rangle=\mathrm{PG}(4, q)$ ).

We now claim that $\iota$ is the identity. Indeed, if not, then let $K_{4}$ belong to a parallel class which is paired with the parallel class containing $K_{5}$. Let $K_{4}^{\prime}$ be a block parallel to $K_{4}$ but not containing $x$. Project $\mathcal{P} \backslash\{x\}$ from $x$ onto a 3 -space $\Sigma^{*}$ not containing $x$. The projections of $K_{5} \backslash\{x\}$ and $K_{4}^{\prime}$ are contained in a plane; the projection of $K_{5} \backslash\{x\}$ generates a line $T_{4}$ which is tangent to the $q$-arc $C$, which denotes the projection of $K_{4}^{\prime}$. Let $i \in\{1,2,3\}$. Let $A$ and $B$ denote the projections of $\alpha \backslash\{x\}$ and $\beta \backslash\{x\}$, respectively. Let $N_{i}$ be the line generated by the projection of $K_{i} \backslash\{x\}, i \in\{1,2,3,6,7, \ldots, q+1\}$. Then $N_{i}$ intersects the skew lines $A, B$ and also the $q$-arc $C$; hence $N_{i}$ is completely determined by $A, B$ and $N_{i} \cap C$. It follows that the plane spanned by $N_{i} \cap A$ and $B$ intersects $C$ in a tangent $T_{i}, i=1,2,3$ (indeed, otherwise some $N_{j}, j \neq i$, meets $N_{i}$ in a point and so the parallel class of $K_{i}$ is paired with the parallel class of $K_{j}$, a contradiction). Hence there are 4 tangent lines $T_{1}, T_{2}, T_{3}, T_{4}$ to $C$ through the point $B \cap\langle C\rangle$. This implies that $B \cap\langle C\rangle$ extends $C$ to a $(q+1)$-arc, see Section 10 of [1]. Similarly, also $A \cap\langle C\rangle$ extends $C$ to a $(q+1)$-arc. It follows that $q$ is even and both points extend $C$ to a hyperoval. But this contradicts the fact that $T_{4}$ contains a point of $C$, and also contains $A \cap\langle C\rangle$ and $B \cap\langle C\rangle$. Hence $\iota$ is the identity.

It now also follows that $\alpha \cup \beta$ is the union of the tangent lines at $x$ to the $q$-arcs of $\mathcal{B}$ through $x$. Now we again consider the projection from $x$ onto $\Sigma^{*}$, as in the previous paragraph. The arguments there now imply that the projections of $K_{4} \backslash\{x\}$ and $K_{4}^{\prime}$ are contained in a plane, and, with the same notation as above, the points $A \cap\langle C\rangle$ and $B \cap\langle C\rangle$ extend $C$ to a hyperoval (hence $q$ is even). In $\operatorname{PG}(4, q)$, this means that the intersections $a_{4}$ and $b_{4}$ of the tangents at $x$ to $K_{4}$ with $\left\langle K_{4}\right\rangle \cap\left\langle K_{4}^{\prime}\right\rangle$ extend $K_{4}^{\prime}$ to a hyperoval. Now interchange the roles of $K_{4}$ and $K_{4}^{\prime}$, where $x$ is interchanged with some $x^{\prime} \in K_{4}^{\prime}$. Then the tangents at $x^{\prime}$ of $K_{4}^{\prime}$ are precisely the lines $\left\langle x^{\prime}, a_{4}\right\rangle$ and $\left\langle x^{\prime}, b_{4}\right\rangle$, and they must intersect $\left\langle K_{4}\right\rangle \cap\left\langle K_{4}^{\prime}\right\rangle=\left\langle a_{4}, b_{4}\right\rangle$ in the points extending $K_{4}$ to a hyperoval. It follows that $a_{4}$ and $b_{4}$ extend $K_{4}$ to a hyperoval, and hence they extend every block parallel to $K_{4}$ to a hyperoval. Hence $a_{4}$ and $b_{4}$ only depend on the parallel class $\mathfrak{P}_{4}$ containing $K_{4}$, and 
we can do this for the parallel class $\mathfrak{P}_{i}, i=1,2, \ldots, q+1$, containing any $K_{i}$, obtaining the points $a_{i}, b_{i}$. We may assume that all $a_{i} \in \alpha$ and all $b_{i} \in \beta$. By varying the point $x$, we see that all $a_{i}$ form a unique line $A^{*}$ and all $b_{i}$ form a unique line $B^{*}$. If we add either $A^{*}$ or $B^{*}$ to $\mathcal{P}$, and define new blocks as the ovals arising from the old blocks by adding the appropriate $a_{i}$ or $b_{i}$, respectively, and also add the appropriate block $A^{*}$ or $B^{*}$, then we obtain a projective plane. By deleting an arbitrary block which is an oval, we have a representation of an affine plane in $\mathrm{PG}(4, q)$ where all lines are $q$-arcs, except for one, which is an affine line. This brings us back to the situation of Paragraph 2.2.2, and we obtain Case $(c 3)$ of the Main Result-Affine Planes. Note that by the last part of Paragraph 2.2.2 Condition (iii) of the Main Result- Affine Planes is satisfied in Case (c3).

\section{There are at most two self-pairings}

Since we assume $q \geq 7$, and there are $q+1$ parallel classes, there are at least three nonidentity pairings. Suppose that the distinct parallel classes $\mathfrak{P}_{1}$ and $\mathfrak{P}_{2}$ are paired with each other. From the previous considerations we know that every member of $\mathfrak{P}_{1}$ together with every member of $\mathfrak{P}_{2}$ generates a 3 -space, and that any two members of either $\mathfrak{P}_{1}$ or $\mathfrak{P}_{2}$ generate the whole 4-space. If we consider the corresponding sets $\mathfrak{V}_{1}$ and $\mathfrak{V}_{2}$ of planes generated by the blocks of $\mathfrak{P}_{1}$ and $\mathfrak{P}_{2}$, respectively, and we dualize to sets $\mathfrak{L}_{1}$ and $\mathfrak{L}_{2}$ of $q$ lines, respectively, then we see that $\mathfrak{L}_{1}$ and $\mathfrak{L}_{2}$ each consists of $q$ members of a system of generators of a common hyperbolic quadric in some 3 -space. The dual of this 3 -space is the point $p$ contained in all members of $\mathfrak{V}_{1} \cup \mathfrak{V}_{2}$.

Suppose, by way of contradiction, that $p, u, v$, with $u, v \in \mathcal{P}, u \neq v$, are contained in a line. Clearly $p \notin \mathcal{P}$. Hence the line $\langle u, v\rangle$ is contained in the planes of $\mathfrak{V}_{1}$ and $\mathfrak{V}_{2}$ that contain either $u$ or $v$ and $u$ and $v$ are contained in the same member of $\mathfrak{V}_{1}$ and in the same member of $\mathfrak{V}_{2}$, clearly a contradiction, as a block can not belong to both $\mathfrak{P}_{1}$ and $\mathfrak{P}_{2}$.

Hence $p$ extends all members of $\mathfrak{P}_{1} \cup \mathfrak{P}_{2}$ to ovals. If we project $\mathcal{P}$ from $p$ onto some 3 -space $\Sigma$ not containing $p$, then we obtain $q^{2}$ points of a hyperbolic quadric $\mathcal{H}$, lying on $2 q$ lines. The unique point of $\mathcal{H}$ not covered by these lines will be denoted by $r$. Let $K$ be any block not belonging to $\mathfrak{P}_{1} \cup \mathfrak{P}_{2}$. Its projection $K^{\prime}$ is contained in $\mathcal{H}$ and contains a point of every generator containing the projection of a member of $\mathfrak{P}_{1}$, and similarly for $\mathfrak{P}_{2}$. It follows that $K^{\prime}$ is contained in a conic $C^{\prime}$ which necessarily contains $r$. Since there are $q^{2}-q$ conics on $\mathcal{H}$ containing $r$, we see that there is a bijective correspondence between the members of $\mathcal{B} \backslash\left(\mathfrak{P}_{1} \cup \mathfrak{P}_{2}\right)$ and the conics on $\mathcal{H}$ through $r$. Suppose now that $K$ belongs to a parallel class $\mathfrak{P}_{3}$ which is paired with $\mathfrak{P}_{4} \neq \mathfrak{P}_{3}$. Let $p^{*}$ be the intersection 
of the planes of the members of $\mathfrak{P}_{3} \cup \mathfrak{P}_{4}$, and let $p^{\prime}$ be the projection of $p^{*}$. Since there are $2 q>q+1$ projections of such planes, not all these projections can contain a common line, and so $p^{\prime}$ coincides with $r$. Interchanging the roles of $\mathfrak{P}_{1} \cup \mathfrak{P}_{2}$ and $\mathfrak{P}_{3} \cup \mathfrak{P}_{4}$, we see that $K_{i} \cup\{p\}$ is a conic for all $K_{i} \in \mathfrak{P}_{i}, i=1,2$.

Now let $\left(\mathfrak{P}_{5}, \mathfrak{P}_{6}\right)$ be a third pair of distinct parallel classes paired to each other, $\mathfrak{P}_{5}, \mathfrak{P}_{6} \notin$ $\left\{\mathfrak{P}_{1}, \mathfrak{P}_{2}, \mathfrak{P}_{3}, \mathfrak{P}_{4}\right\}$ and let $K_{i} \in \mathfrak{P}_{i}, i=5,6$. By re-choosing $\Sigma$ we may suppose that $r$ extends $K_{i}$ to a conic $C_{i}, i=5,6$. Moreover, since $K_{5} \cup K_{6}$ generates a 3 -space (as $\mathfrak{P}_{5}$ is paired with $\mathfrak{P}_{6}$ ), we may even assume that $\Sigma=\left\langle K_{5}, K_{6}\right\rangle$. Projecting $\mathcal{P}$ from $p^{*}$ onto $\Sigma$ yields a hyperbolic quadric $\mathcal{H}^{*}$. Clearly, $\left(C_{5} \cup C_{6}\right) \subseteq \mathcal{H} \cap \mathcal{H}^{*}$, so both $\mathcal{H}$ and $\mathcal{H}^{*}$ belong to the pencil $\mathfrak{B}$ of quadrics in $\Sigma$ with base $C_{5} \cup C_{6}$.

Since $K_{5}$ and $K_{6}$ belong to different parallel classes, they meet in some point $r^{\prime}$. We now coordinatize the situation using coordinates $\left(X_{0}, X_{1}, X_{2}, X_{3}, X_{4}\right)$. Let $\Sigma$ be determined by the equation $X_{4}=0$. Choose $r=(1,0,0,0,0)$ and $r^{\prime}=(0,1,0,0,0)$. Let $\left\langle C_{5}\right\rangle$ have equations $X_{3}=X_{4}=0$ and let $\left\langle C_{6}\right\rangle$ have equations $X_{2}=X_{4}=0$. We can choose the coordinates of the intersection of the tangent lines of $C_{5}$ at $r$ and $r^{\prime}$ equal to $(0,0,1,0,0)$, and the coordinates of the intersection of the tangent lines of $C_{6}$ at $r$ and $r^{\prime}$ equal to $(0,0,0,1,0)$. Furthermore, we can assume that the point $(1,1,1,0,0)$ belongs to $C_{5}$. This pins down the equations of $K_{5}$ to $X_{3}=X_{4}=X_{0} X_{1}-X_{2}^{2}=0$, whereas the equations of $K_{6}$ are $X_{2}=X_{4}=X_{0} X_{1}-\rho X_{3}^{2}$, for some $\rho \in \mathrm{GF}(q) \backslash\{0\}$. By choosing the point $(1,1,1,1,0)$ appropriately, we may assume that $\rho$ is either 1 , or a given non-square $\rho_{0}$ of $\operatorname{GF}(q)$; if $q$ is even then we may assume that $\rho=1$, if $q$ is odd, then we may assume that $\rho \in\left\{1, \rho_{0}\right\}$. It follows that the equations of an arbitrary member $Q_{\alpha}, \alpha \in \operatorname{GF}(q) \cup\{\infty\}$, of the pencil $\mathfrak{B}$ are

$$
X_{4}=X_{2}^{2}-X_{0} X_{1}+\rho X_{3}^{2}+\alpha X_{2} X_{3}=0 .
$$

Note that the point $r$ belongs to every member of the pencil, and that the tangent plane at $r$ to $Q_{\alpha}$, for all $\alpha \in \mathrm{GF}(q)$, has equations $X_{1}=X_{4}=0$. It follows that $Q_{\alpha}$ is hyperbolic if and only if the intersection of $Q_{\alpha}$ with that tangent plane is the union of two distinct lines, i.e., the equation $X_{2}^{2}+\alpha X_{2}+\rho=0$ has exactly two (different) solutions.

Suppose $\mathcal{H}=Q_{\theta}$ and $\mathcal{H}^{*}=Q_{\theta^{*}}, \theta, \theta^{*} \in \mathrm{GF}(q)$. We can assign the coordinates $(0,0,0,0,1)$ to $p$ and $(1,0,0,0,1)$ to $p^{*}$. Then $\mathcal{P}$ is contained in the cone $\overline{\mathcal{H}}$ with vertex $p$ and base $\mathcal{H}$, and also in the cone $\overline{\mathcal{H}}^{*}$ with vertex $p^{*}$ and base $\mathcal{H}^{*}$. Hence $\mathcal{P}$ is contained in the intersection $\overline{\mathcal{H}} \cap \overline{\mathcal{H}}^{*}$, which has equations

$$
\left\{\begin{array}{l}
0=X_{2}^{2}-X_{0} X_{1}+\rho X_{3}^{2}+\theta X_{2} X_{3} \\
0=X_{2}^{2}-\left(X_{0}-X_{4}\right) X_{1}+\rho X_{3}^{2}+\theta^{*} X_{2} X_{3}
\end{array}\right.
$$

Now, we also know that no point of $\mathcal{P}$ is projected onto the lines of $\mathcal{H}$ through $r$, and one verifies that these points are characterized by the fact that their coordinate $X_{1}$ is equal 
to 0 . Hence we may assume that every point of $\mathcal{P}$ has $X_{1}$-coordinate equal to 1 . We can parameterize such points on $\overline{\mathcal{H}}$ using the parameters $a, b, c \in \mathrm{GF}(q)$ as follows:

$$
\left\{\begin{array}{l}
X_{1}=1, \\
X_{2}=a, \\
X_{3}=b, \\
X_{4}=c, \\
X_{0}=a^{2}+\rho b^{2}+\theta a b .
\end{array}\right.
$$

Likewise, we can parameterize such points on $\overline{\mathcal{H}}^{*}$, using the same parameters $a, b, c \in$ $\mathrm{GF}(q)$, anticipating on taking the intersection, as follows:

$$
\left\{\begin{array}{l}
X_{1}=1 \\
X_{2}=a \\
X_{3}=b \\
X_{4}=c \\
X_{0}=c+a^{2}+\rho b^{2}+\theta^{*} a b .
\end{array}\right.
$$

Hence a parameterization of the intersection is given by

$$
\left\{\begin{array}{l}
X_{1}=1 \\
X_{2}=a \\
X_{3}=b \\
X_{4}=\left(\theta-\theta^{*}\right) a b \\
X_{0}=a^{2}+\rho b^{2}+\theta a b .
\end{array}\right.
$$

Since the parameters can take $q^{2}$ different values, this intersection coincides with $\mathcal{P}$.

Now, if $\theta=\theta^{*}$, then $\mathcal{P}$ is contained in the 3 -space with equation $X_{4}=0$, a contradiction. Hence we can recoordinatize as follows: $X_{i}^{\prime}=X_{i}$, for $i=1,2,3, X_{4}^{\prime}=X_{4} /\left(\theta-\theta^{*}\right)$ and $X_{0}^{\prime}+\theta X_{4}^{\prime}=X_{0}$. Consequently we see that the set $\mathcal{P}$ is projectively equivalent with the following set of points:

$$
\left\{\begin{array}{l}
X_{1}=1 \\
X_{2}=a \\
X_{3}=b \\
X_{4}=a b \\
X_{0}=a^{2}+\rho b^{2}
\end{array}\right.
$$

with $a, b \in \mathrm{GF}(q)$, and which is independent of $\theta$ and $\theta^{*}$. Since $\rho=1$ if $q$ is even, we have a unique solution in this case, and this must coincide with Case $(c 2)$ in the Main 
Result-Affine Planes. If $q$ is odd, then $\rho \in\left\{1, \rho_{0}\right\}$, yielding at most two different cases; hence there are precisely two different cases, which are described in Case $(c 2)$ of the Main Result-Affine Planes.

This completes the case $d=4$.

\subsection{Case $d=3$}

We continue to assume $q \geq 7$.

\subsubsection{All blocks are $q$-arcs}

In this case, $\mathcal{P}$ is a $q^{2}$-cap of $\mathrm{PG}(3, q)$. Any such cap is, for $q>2$, contained in an ovoid $\mathcal{O}$ (see Sections 18.3, 18.4 and 18.5 of [2]). Let $\mathcal{O}=\mathcal{P} \cup\{p\}$. By Assumption (iii), the planes generated by the blocks must contain $p$. Hence we obtain Case (b1). Clearly, there is no extension to a projective plane.

\subsubsection{There is a point $p \in \mathcal{P}$ such that at most two blocks in $\mathcal{B}$ through $p$ are $q$-arcs}

In this case, we claim that all blocks through $p$ are affine lines. Indeed, suppose not and let $K$ be a $q$-arc in $\mathcal{B}$ containing $p$. Let $L_{1}, L_{2}, L_{3} \in \mathcal{B}$ be three affine lines through $p$. If $K^{\prime} \neq K$ is some block parallel to $K$, then $K^{\prime}$ intersects each of $L_{1}, L_{2}, L_{3}$. If $K^{\prime}$ is an affine line, then $\left\langle L_{1}, L_{2}, L_{3}\right\rangle$ is a plane and we see that this plane must contain $\mathcal{P}$, a contradiction. Hence every triple of affine lines of $\mathcal{B}$ through $p$ generates $\mathrm{PG}(3, q)$, and every block not through $p$ is a $q$-arc.

Suppose first that there is another block through $p$ that is a $q$-arc, say $J, J \neq K$. Let $L_{1}, L_{2}, \ldots, L_{q-1}$ be the blocks through $p$ that are affine lines. By considering a block parallel to $K$, but distinct from $K$, we see that there exists a line of $\mathrm{PG}(3, q)$ through $p$ which is not contained in a plane $\left\langle L_{i}, L_{j}\right\rangle, i, j \in\{1,2, \ldots, q-1\}, i \neq j$. Then by Section 10.3 of [1], there are precisely three (when $q$ is even) or two (when $q$ is odd) lines $M_{1}, M_{2}$ and possibly $M_{3}$ through $p$ such that no such line lies in a plane together with two lines of the set $\left\{\left\langle L_{1}\right\rangle,\left\langle L_{2}\right\rangle, \ldots,\left\langle L_{q-1}\right\rangle\right\}$. These two or three lines are the only lines through $p$ which are not contained in a plane $\left\langle L_{i}, L_{j}\right\rangle, i, j \in\{1,2, \ldots, q-1\}, i \neq j$. If $q$ is odd, we put $M_{3}=M_{2}$. Let $S \in \mathcal{B}$ be any block parallel to $K$ but distinct from $K$. Then it is clear that the point $J \cap S$ must be found among the points $\langle S\rangle \cap\left(M_{1} \cup M_{2} \cup M_{3}\right)$. 
Varying $S$, this implies that $J$ is contained in the union of at most three lines and must hence contain a triple of collinear points, a contradiction. Hence $p$ is contained in $q$ affine lines $L_{1}, L_{2}, \ldots, L_{q}$ of $\mathcal{B}$. Let $M_{2}, M_{3}$ be the lines of $\mathrm{PG}(3, q)$ through $p$ not contained in any plane $\left\langle L_{i}, L_{j}\right\rangle, i, j \in\{1,2, \ldots, q\}, i \neq j$, where $M_{2}=M_{3}$ for $q$ odd and $M_{2} \neq M_{3}$ for $q$ even. Now let $C$ be an arbitrary block not parallel to $K$, and not through $p$. Then, as above, the point $C \cap K$ must be found among $\langle C\rangle \cap M_{2}$ and $\langle C\rangle \cap M_{3}$. Hence $K$ is contained in the union of at most two lines, a contradiction. This implies that $K=L_{q+1}$ is also an affine line, proving our claim.

Let $v_{i}$ be the point of $\left\langle L_{i}\right\rangle$ which does not belong to $L_{i}, i=1,2, \ldots, q+1$. The planes of the $q-1$ blocks parallel to $L_{i}$ clearly contain $v_{i}, i=1,2, \ldots, q+1$; adding $v_{i}$ to these blocks produces $q-1$ ovals through $v_{i}$. As parallel blocks do not intersect, all these ovals have a common tangent line at $v_{i}, i=1,2, \ldots, q+1$. Let $C$ be one of those ovals (through $v_{i}$, for some $\left.i \in\{1,2, \ldots, q+1\}\right)$. Let $s \in C \backslash\left\{v_{i}\right\}$. Since different blocks through $s$ do not intersect in more than one point, and since the planes of such blocks do not share a point of type $v_{j}, j \in\{1,2, \ldots, q+1\}$ (as otherwise they are both parallel to $L_{j}$ ), all blocks through $s$ that are $q$-arcs have a common tangent line $T$ at $s$ which is also tangent to the $q$ corresponding ovals.

Let $O_{1}, O_{2}, O_{3}$ be three of the $q^{2}-1$ ovals obtained from the $q$-arcs in $\mathcal{B}$ by adding some member of $\left\{v_{1}, \ldots, v_{q+1}\right\}$, and suppose $O_{1} \cap O_{2}=\left\{s_{3}\right\} \neq\left\{s_{2}\right\}=O_{1} \cap O_{3} \neq O_{2} \cap O_{3}=$ $\left\{s_{1}\right\} \neq\left\{s_{3}\right\}$. The three tangents at $s_{1}, s_{2}, s_{3}$ are pairwise contained in the same plane, but are not all contained in a common plane; hence they have a point $n$ in common. Hence all tangents of the $q^{2}-1$ ovals obtained from the $q$-arcs in $\mathcal{B}$ contain $n$. It follows that $q$ is even. Considering the $q+1$ planes $\left\langle n, L_{i}\right\rangle, i=1,2, \ldots, q+1$, and the $q^{2}-1$ planes of the ovals, there arise $q^{2}+q$ planes through $n$ none of which contains two points of $\left\{v_{1}, v_{2}, \ldots, v_{q+1}\right\}$. It follows that the remaining plane through $n$ contains $v_{1}, v_{2}, \ldots, v_{q+1}$. Clearly $\left\{v_{1}, v_{2}, \ldots, v_{q+1}\right\}$ is an oval with nucleus $n$.

This is Case (b4) of Main Result-Affine Planes. Clearly it uniquely extends to Case (B) of Main Result-Projective planes.

\subsubsection{There is some affine line in $\mathcal{B}$ and every point $p \in \mathcal{P}$ is contained in at least three $q$-arcs belonging to $\mathcal{B}$}

Let $x \in \mathcal{P}$. By assumption, there are at least three $q$-arcs contained in $\mathcal{B}$ through $x$. Suppose first that there are at least four such $q$-arcs $K_{1}, K_{2}, K_{3}, K_{4}$ through $x$. We claim that these have a common tangent line at $x$. Indeed, let $L_{i}^{(1)}$ and $L_{i}^{(2)}, i=1,2,3,4$, be the tangent lines at $x$ to $K_{i}$. By Assumption (iii), the line $\left\langle K_{i}\right\rangle \cap\left\langle K_{j}\right\rangle, i, j \in\{1,2,3,4\}$, 
$i \neq j$, is tangent to both $K_{i}$ and $K_{j}$. Hence $\left|\left\{L_{i}^{(1)}, L_{i}^{(2)}\right\} \cap\left\{L_{j}^{(1)}, L_{j}^{(2)}\right\}\right|=1$, for all $i, j \in\{1,2,3,4\}, i \neq j$. It is easy to see that this implies our claim. Now suppose that there are exactly three $q$-arcs of $\mathcal{B}$ through $x$. Then there are at least three affine lines of $\mathcal{B}$ containing $x$; by Assumption (iii) any three such lines generate PG $(3, q)$. Let $K$ be any of the three $q$-arcs. Then the three planes $\pi_{i}, 1=1,2,3$, obtained by joining any two of the three affine lines pairwise intersect $\langle K\rangle$ in three distinct lines $L_{i}, i=1,2,3$, respectively; hence one of these lines, say $L_{1}$, intersects $K$ in a further point $y$. Joining $y$ with a point $x_{1}$ of one of the affine lines in $\pi_{1}$ such that the line $\left\langle y, x_{1}\right\rangle$ intersects the other affine line of $\pi_{1}$ nontrivially, we see that Assumption (iii) and Assumption (ii) imply that the block containing $y$ and $x_{1}$ is an affine line. This then readily implies that $\mathcal{P}$ is contained in $\pi_{1}$, a contradiction. In conclusion, we have shown that the $q$-arcs of $\mathcal{B}$ through a fixed point $x \in \mathcal{P}$ all admit a common tangent $T_{x}$ at $x$.

Note that the arguments in the previous paragraph imply that through any point of $\mathcal{P}$ pass at most two affine lines belonging to $\mathcal{B}$.

Now let $K \in \mathcal{B}$ be a fixed $q$-arc, and let $x \in \mathcal{P} \backslash K$. By the previous paragraph, there are at least three $q$-arcs $K_{1}, K_{2}, K_{3}$ belonging to $\mathcal{B}$ through $x$ intersecting $K$ in points, say $x_{1}, x_{2}, x_{3}$, respectively. The common tangent $T_{x}$ and the tangent line at $x_{i}$ to $K_{i}$, say $T_{x_{i}}$, are contained in the same plane $\left\langle K_{i}\right\rangle, i=1,2,3$, and hence meet in some point $y_{i}$. If $y_{1} \neq y_{2}$, then $T_{x} \subseteq\langle K\rangle$ and so $\langle K\rangle=\left\langle K_{1}\right\rangle=\left\langle K_{2}\right\rangle$, a contradiction. Consequently, $T_{x}, T_{x_{1}}, T_{x_{2}}, T_{x_{3}}$ intersect in a common point $n$. Since we can also first choose $x_{1}$ and $x_{2}$, and can find a corresponding $x \in \mathcal{P}$ outside $K$, we conclude that all common tangents contain the point $n$. This point $n$ extends all $q$-arcs of $\mathcal{B}$ to ovals.

Assume, by way of contradiction, that three affine lines $L, L^{\prime}, L^{\prime \prime} \in \mathcal{B}$ belong to different parallel classes and have no point in common. Then it is easy to see that $\left\langle L, L^{\prime}, L^{\prime \prime}\right\rangle$ is a plane containing $\mathcal{P}$, a contradiction. Also the same argument implies that, if $L, L^{\prime}, L^{\prime \prime}$ are three affine lines of $\mathcal{B}$ with $L$ parallel to $L^{\prime}$, but not with $L^{\prime \prime}$, then $\langle L\rangle$ and $\left\langle L^{\prime}\right\rangle$ are skew lines in $\mathrm{PG}(3, q)$.

By assumption, there is a block of $\mathcal{A}$ which is an affine line. Let $L_{1} \in \mathcal{B}$ be an affine line.

Suppose $\left\{L_{1}, L_{2}, \ldots, L_{q}\right\}$ is the parallel class of blocks of $\mathcal{A}$ containing $L_{1}$, with $L_{1}, \ldots, L_{k}$ affine lines and $L_{k+1}, \ldots, L_{q} q$-arcs, $1 \leq k \leq q$. Put $\left\{\ell_{i}\right\}=\left\langle L_{i}\right\rangle \backslash L_{i}, i=1,2, \ldots, k$. Then $\ell_{i} \in\left\langle L_{j}\right\rangle$ and $n \in\left\langle L_{j}\right\rangle$, for all $i=1,2, \ldots, k$ and all $j=k+1, \ldots, q$.

First, we assume $1<k<q$.

Clearly, $n \neq \ell_{i}$, for any $i=1,2, \ldots, k$, since the plane generated by a $q$-arc belonging to $\mathcal{B}$ and not parallel to $L_{i}$ would then contain $L_{i}$, a contradiction. If the line $\left\langle n, \ell_{i}\right\rangle$, for some $i \in\{1,2, \ldots, k\}$, would contain a point $y$ of $L_{j}$, for some $j \in\{k+1, \ldots, q\}$, 
then the plane spanned by any block through $y$, distinct from $L_{j}$, which is a $q$-arc, would contain $L_{i}$ (and such a block exists by our assumption), a contradiction. It follows that the points $n, \ell_{1}, \ldots, \ell_{k}$ are collinear and that the ovals $L_{k+1} \cup\{n\}, \ldots, L_{q} \cup\{n\}$ have a common tangent $\left\langle\ell_{1}, n\right\rangle$ at $n$.

Assume that $\ell_{1}=\ell_{2} \neq \ell_{3}$. Let $z$ be the intersection of $\left\langle L_{1}, L_{2}\right\rangle$ and $L_{3}$, and choose $v \in L_{1}$ and $w \in L_{2}$ such that $v, w, z$ are collinear. If the block $Z$ through $w$ and $v$ is an affine line, then we see that $\mathcal{P}$ is contained in the plane $\left\langle L_{1}, L_{2}\right\rangle$, a contradiction. If $Z$ is a $q$-arc and if $z \neq \ell_{3}$, then we obtain a contradiction to Assumption (iii). If $Z$ is a $q$-arc and $z=\ell_{3}$, then $\langle Z\rangle$ contains $n, \ell_{1}, \ell_{3}$, and so $\left\langle L_{1}, L_{2}\right\rangle$ contains $Z$, also a contradiction. Hence either all points $\ell_{1}, \ldots, \ell_{k}$ are distinct and collinear, or they all coincide.

First, let $\ell_{1}, \ldots, \ell_{k}$ be distinct collinear points. Put $L_{k+1}=\left\{x_{1}, x_{2}, \ldots, x_{q}\right\}$. Let $\bar{N}_{i}$ be the line of $\mathrm{PG}(3, q)$ which contains $x_{i}$ and intersects $L_{1}$ and $L_{2}, i=1,2, \ldots, q$. By Assumption $(i i i), \bar{N}_{i}$ contains an affine line $N_{i}$ which belongs to $\mathcal{B}, i=1,2, \ldots, q$. The blocks $N_{1}, N_{2}, \ldots, N_{q}$ clearly belong to a common parallel class of $\mathcal{A}$. As $q \geq 7$, there is a line $\bar{M}$ of $\mathrm{PG}(3, q)$ distinct from $L_{1}, L_{2}$ which intersects the affine lines $N_{1}, N_{2}, N_{3}$ nontrivially. Assumption (iii) again implies that $\bar{M}$ contains an affine line $M \in \mathcal{B}$, which is clearly parallel to $L_{1}$ and hence belongs to $\left\{L_{3}, \ldots, L_{k}\right\}$. Hence $k \geq 3$. It follows that $\left\langle L_{1}\right\rangle,\left\langle L_{2}\right\rangle, \ldots,\left\langle L_{k}\right\rangle, \bar{N}_{1}, \ldots, \bar{N}_{q},\left\langle\ell_{1}, \ell_{2}\right\rangle$ are generators of a hyperbolic quadric $\mathcal{H}$. But $\ell_{1}, \ldots, \ell_{k}$ and $L_{i}, i \in\{k+1, \ldots, q\}$, belong to a common plane and are contained in $\mathcal{H}$, a contradiction.

Hence $\ell_{1}=\ell_{2}=\cdots=\ell_{k}=: \ell$. Since $k \geq 2$, we immediately deduce that every block not parallel to $L_{1}$ is a $q$-arc. We claim that $\mathcal{K}=L_{k+1} \cup \cdots \cup L_{q}$ is a cap. Indeed, if not, then we find three points $x, y, z \in \mathcal{K}$ collinear in $\mathrm{PG}(3, q)$. By Condition (iii), these must be contained in an affine line $W \in \mathcal{B}$, contradicting the fact that it must be a $q$-arc. Our claim is proved, and $\mathcal{K}$ is a $\left(q^{2}-k q\right)$-cap of $\mathrm{PG}(3, q)$, which clearly has no point in common with $\left\langle L_{1}, L_{i}\right\rangle$, for all $i \in\{2,3, \ldots, k\}$. Let $\pi$ be a plane of $\mathrm{PG}(3, q)$ containing $L_{1}$ but not $L_{i}$, for all $i \in\{2,3, \ldots, k\}$. If $\pi \cap \mathcal{K}$ contains at least two points $x, y$, then Condition (iii) implies that $\langle x, y\rangle$ intersects $\left\langle L_{1}\right\rangle$ in $\ell$ (remember that the block through $x$ and $y$ is a $q$-arc; in particular no further point of $\mathcal{P}$ lies on $\langle x, y\rangle)$. Hence $\pi \cap \mathcal{K}$ contains at most two elements. Further, we claim that the plane $\left\langle L_{1}, n\right\rangle$ does not contain any element of $\mathcal{P}$. Indeed, suppose $x \in \mathcal{P} \backslash L_{1}$ is contained in $\left\langle L_{1}, n\right\rangle$. Then any block of $\mathcal{A}$ through $x$ not parallel to $L_{1}$ is a $q$-arc $K^{\prime}$ such that $\left\langle K^{\prime}\right\rangle$ contains $n$ and also $\ell$, and hence also every $\left\langle L_{i}\right\rangle$ as it intersects each $L_{i}$ nontrivially, $i=1,2, \ldots, k$, a contradiction. Our claim follows. 
It now follows that $q^{2}-k q=|\mathcal{K}| \leq 2(q-k+1)$, which means

$$
k \geq q-\frac{2}{q-2} .
$$

Since $q \geq 7$, this implies that $k=q$, a contradiction.

Hence it follows that, if $\mathcal{B}$ contains two parallel affine lines $L_{1}, L_{2}$, then all blocks parallel to $L_{1}$ are affine lines. Suppose $\left\{L_{1}, L_{2}, \ldots, L_{q}\right\}$ is a parallel class of $\mathcal{A}$ and all of $L_{1}, \ldots, L_{q}$ are affine lines. Put $\left\{\ell_{i}\right\}=\left\langle L_{i}\right\rangle \backslash L_{i}, i=1,2, \ldots, q$. As in the case $1<k<q$ it is shown that $n \neq \ell_{i}$, with $i=1,2, \ldots, q$. First assume that $\ell_{1}=\ell_{2}=\cdots=\ell_{q}=: \ell$. Then $n \notin\left\langle L_{i}, L_{j}\right\rangle, i, j \in\{1,2, \ldots, q\}, i \neq j$, as otherwise $\mathcal{P}$ is contained in the plane $\left\langle L_{i}, L_{j}\right\rangle$. Clearly we obtain Case (b2) of Main Result-Affine Planes. For $q$ even, this model extends to Case $(B)$ of Main result-Projective Planes.

Next assume that $\ell_{1} \neq \ell_{2}$. Assume by way of contradiction that not all points $\ell_{1}, \ldots, \ell_{q}$ are distinct; then without loss of generality we may assume $\ell_{1}=\ell_{q}$. This already implies that all blocks not parallel to $L_{1}$ are $q$-arcs. Condition (iii) implies that the intersection point $r$ of $\left\langle L_{2}\right\rangle$ and $\left\langle L_{1}, L_{q}\right\rangle$ coincides with $\ell_{2}$. Choose two points $y_{1} \in L_{1}$ and $y_{q} \in L_{q}$ such that $y_{1}, y_{q}, \ell_{2}$ are collinear. Let $K$ be the block through $y_{1}$ and $y_{q}$. Then $\langle K\rangle$ intersects $L_{2}$ in a point off $\left\langle L_{1}, L_{q}\right\rangle$, and contains $\ell_{2}$, hence $\langle K\rangle$ contains $L_{2}$, a contradiction. Consequently, $\ell_{1}, \ell_{2}, \ldots, \ell_{q}$ are distinct.

Consider two lines $\bar{M}_{1}, \bar{M}_{2}$ of $\mathrm{PG}(3, q)$ intersecting $L_{1}, L_{2}, L_{3}$ (as affine lines; this is possible since $q \geq 7$ ). As before, Assumption (iii) implies that $\bar{M}_{1}, \bar{M}_{2}$ are the projective completions of affine lines $M_{1}, M_{2}$ belonging to $\mathcal{B}$, and necessarily parallel in $\mathcal{A}$. By the foregoing, all members of the parallel class of $M_{1}$ are affine lines $M_{1}, M_{2}, \ldots, M_{q}$. Set $m_{i}=\left\langle M_{i}\right\rangle \backslash M_{i}, i=1,2, \ldots, q$. Clearly, the lines $\left\langle L_{1}\right\rangle,\left\langle L_{2}\right\rangle, \ldots,\left\langle L_{q}\right\rangle,\left\langle M_{1}\right\rangle, \ldots,\left\langle M_{q}\right\rangle$ are generators of a hyperbolic quadric $\mathcal{H}$, the points $\ell_{1}, \ldots, \ell_{q}$ are on a line $L$ of PG $(3, q)$, and the points $m_{1}, \ldots, m_{q}$ are on a line $M$ of $\mathrm{PG}(3, q)$. As a $q$-arc of $\mathcal{B}$ intersects all $L_{i}$ and $M_{i}, i=1,2, \ldots, q$, it must intersect $L$ and $M$ in $L \cap M$. Hence $L \cap M=\{n\}$. It is now clear that we obtain Case (b3) of Main Result-Affine Planes. Clearly, this model cannot be extended to a projective plane.

Hence we now only have to deal with the case that no parallel class of blocks of $\mathcal{A}$ contains at least two affine lines, but there exists at least one affine line $L$ in $\mathcal{B}$ (the case $k=1$ ). Note that there is at most one other affine line $M$ in $\mathcal{B}$, which meets $L$ in a point $x \in \mathcal{P}$. If $M$ does not exist, then, as before in the case $k>1$ and $\ell_{1}=\ell_{2}=\cdots=\ell_{k}$, one shows that the $q$ blocks parallel to $L$ are affine lines, a contradiction.

So we may assume that $M$ exists. As in the case $k>1$ and $\ell_{1}=\ell_{2}=\cdots=\ell_{k}$ one shows that every plane containing $L$ but not $M$ contains at most two points of $\mathcal{P} \backslash L$. Hence 
$q^{2} \leq(2 q-1)+2 q$ (the $2 q-1$ comes from $L \cup M$, the $2 q$ comes from the $q$ planes through $L$ not containing $M$ ), which implies $q \leq 3$, a contradiction.

This concludes the case $d=3$.

Our main results are proved.

\section{Remark on the small cases and on Condition (iii)}

\subsection{The small cases $q=2,3,4,5$}

The case $q=2$ is trivial.

Now let $\mathcal{P}$ be any cap of size 9 in $\operatorname{PG}(d, 3)$, with $\langle\mathcal{P}\rangle=\operatorname{PG}(d, 3)$ and $d \in\{3,4, \ldots, 8\}$. Then any bijection of $\operatorname{AG}(2,3)$ onto $\mathcal{P}$ yields a representation of $\operatorname{AG}(2,3)$ in $\operatorname{PG}(d, 3)$ satisfying the requirements.

For $q \in\{4,5\}$, any representation of $\mathrm{AG}(2, q)$ in $\mathrm{PG}(d, q)$ satisfying $(i)$ (but without any restriction on $d$ or $q$ ), (ii) and (iii) implies $d \leq 5$. It is certainly possible to handle the remaining cases by hand or by computer, but we have chosen not to do it here to keep the paper at a reasonable length.

\subsection{Condition (iii)}

If $d \geq 5$ then Condition (iii) is always satisfied for $q>3$. Indeed, if some point $p$ would be contained in the plane spanned by some block $L_{1}$, then we can choose blocks $L_{2}$ and $L_{3}$ such that $p \in L_{2}$ and $L_{1}, L_{2}, L_{3}$ pairwise intersect in distinct points. As every point is contained in a block intersecting $L_{1} \cup L_{2} \cup L_{3}$ in three distinct points, we see that $\left\langle L_{1}, L_{2}, L_{3}\right\rangle=\mathrm{PG}(d, q)$, but as $p \in\left\langle L_{1}\right\rangle \cap\left\langle L_{2}\right\rangle,\left\langle L_{1}, L_{2}, L_{3}\right\rangle$ is at most 4-dimensional, a contradiction.

Let $d=2$ and consider a set of $q^{2}+q+1$ conics in $\mathrm{PG}(2, q)$ such that the point set of $\operatorname{PG}(2, q)$ together with these conics form a PG $(2, q)$. Removing one of these conics and all points on it yields a representation of $\mathrm{AG}(2, q)$ in $\mathrm{PG}(2, q)$ satisfying $(i)$ (with $d=2$ and with no restriction on $q)$, (ii), but not satisfying (iii).

Consider $(c 4)$ with $q \neq 2$ in Main Result-Affine Planes and let $p$ be a point of type $\left(a^{\sigma}, b^{\sigma}, c^{\sigma}, a c^{\sigma-1}, b c^{\sigma-1}\right)$ with $b=0$ and $c \neq 0$. Now project $\mathcal{P}$ from $p$ onto a 3 -space 
PG $(3, q)$ not containing $p$. Then there arises a representation of $\operatorname{AG}(2, q)$ in $\operatorname{PG}(3, q)$ satisfying $(i)$ (with $d=3$ and $q \neq 2$ ), (ii), but not satisfying (iii).

Consider $(a)$ with $q \neq 2$ in Main Result-Affine Planes. Let $D$ be a conic on $\mathcal{V}_{2}^{4}$, distinct from the removed conic $C$. Consider a 3 -space PG $(3, q)$ of $\mathrm{PG}(5, q)$ which intersects $\mathcal{V}_{2}^{4}$ in $D$ and a point $r$ not on $C$. Let $p$ be a point of $\mathrm{PG}(3, q)$ not in a conic plane of $\mathcal{V}_{2}^{4}$. Now we project $\mathcal{P}$ from $p$ onto a 4 -space $\mathrm{PG}(4, q)$ in $\mathrm{PG}(5, q)$ not containing $p$. Then there arises a representation of $\mathrm{AG}(2, q)$ in $\mathrm{PG}(4, q)$ satisfying $(i)$ (with $d=4$ and $q \neq 2$ ), (ii), but not satisfying (iii).

These examples show that Condition (iii) is necessary and does not follow from the other conditions.

\section{References}

[1] J. W. P. Hirschfeld, Projective Geometries over Finite Fields, 2nd edition, Clarendon Press, Oxford Mathematical Monographs, 1998.

[2] J. W. P. Hirschfeld, Finite Projective Spaces of Three Dimensions, Clarendon Press, Oxford Mathematical Monographs, 1985.

[3] J. W. P. Hirschfeld \& J. A.Thas, General Galois Geometries, Oxford Mathematical Monographs, Oxford Science Publications, The Clarendon Press, Oxford University Press, New York, 1991.

[4] S. E. Payne \& J. A. Thas, Finite Generalized Quadrangles, Research Notes in Mathematics 110, Pitman Advanced Publishing Program, Boston/London/Melbourne, 1984; second edition in EMS Series of Lectures in Mathematics, European Mathematical Society, 2009.

[5] J. A. Thas, K. Thas \& H. Van Maldeghem, Translation Generalized Quadrangles, World Scientific Publishing, Hackensack, 2007.

[6] J. A. Thas \& H. Van Maldeghem, Characterizations of the finite quadric Veroneseans $\mathcal{V}_{n}^{2^{n}}$, Quart. J. Math. 55 (2004), 99-113. 